\title{
Systematic Understanding of Mechanism of Danggui Shaoyao San against Ischemic Stroke Using a Network Pharmacology Approach
}

\author{
Sijie Li, ${ }^{1,2}$ Yong Yang, ${ }^{3}$ Wei Zhang, ${ }^{1,2,3}$ Haiyan Li, ${ }^{1,2}$ Wantong Yu, ${ }^{1,2}$ Chen Gao, ${ }^{1,2}$ Jiali Xu, ${ }^{1,2}$ \\ Wenbo Zhao, ${ }^{1,2}$ and Changhong Ren $\oplus^{1,2}$ \\ ${ }^{1}$ Beijing Key Laboratory of Hypoxia Translational Medicine, Xuanwu Hospital, Capital Medical University, \\ Beijing 100053, China \\ ${ }^{2}$ Center of Stroke, Beijing Institute for Brain Disorder, Capital Medical University, Beijing 100053, China \\ ${ }^{3}$ School of Chinese Medicine, Beijing University of Chines Medicine, Beijing 100029, China
}

Correspondence should be addressed to Changhong Ren; rench@xwhosp.org

Received 8 September 2021; Accepted 7 December 2021; Published 5 January 2022

Academic Editor: Feng Zhang

Copyright $\odot 2022$ Sijie Li et al. This is an open access article distributed under the Creative Commons Attribution License, which permits unrestricted use, distribution, and reproduction in any medium, provided the original work is properly cited.

Purpose. Danggui Shaoyao San (DSS) was developed to treat the ischemic stroke (IS) in patients and animal models. The purpose of this study was to explore its active compounds and demonstrate its mechanism against IS through network pharmacology, molecular docking, and animal experiment. Methods. All the components of DSS were retrieved from the pharmacology database of TCM system. The genes corresponding to the targets were retrieved using OMIM, CTD database, and TTD database. The herbcompound-target network was constructed by Cytoscape software. The target protein-protein interaction network was built using the STRING database. The core targets of DSS were analyzed by Gene Ontology (GO) and Kyoto Encyclopedia of Genes and Genomes (KEGG). Then, we achieved molecular docking between the hub proteins and the key active compounds. Finally, animal experiments were performed to verify the core targets. Triphenyltetrazolium chloride (TTC) staining was used to calculate the infarct size in mice. The protein expression was determined using the Western blot. Results. Compound-target network mainly contained 51 compounds and 315 corresponding targets. Key targets contained MAPK1, SRC, PIK3R1, HRAS, AKT1, RHOA, RAC1, HSP90AA1, and RXRA FN1. There were 417 GO items in GO enrichment analysis $(p<0.05)$ and 119 signaling pathways $(p<0.05)$ in KEGG, mainly including negative regulation of apoptosis, steroid hormone-mediated signaling pathway, neutrophil activation, cellular response to oxidative stress, and VEGF signaling pathway. MAPK1, SRC, and PIK3R1 docked with small molecule compounds. According to the Western blot, the expression of p-MAPK 1, p-AKT, and p-SRC was regulated by DSS. Conclusions. This study showed that DSS can treat IS through multiple targets and routes and provided new insights to explore the mechanisms of DSS against IS.

\section{Introduction}

Stroke has the characteristics of high incidence, high recurrence rate, high fatality rate, high disability rate, and high economic burden, which seriously threatens human health and quality of life. Ischemic stroke (IS) accounts for $70 \%$ to $80 \%$ of all strokes, which results from sudden interruption of the blood supply to areas of the brain [1]. Neurological deficits such as disturbances to consciousness, cognitive and behavioral changes, paralysis, dysphagia, and aphasia are the major sequels of stroke. In China, stroke is the leading cause of death and contributes to a heavy disease burden [2]. The most effective treatment is currently recognized as intravenous thrombolysis and/or endovascular treatment, which prevent irreversible brain tissue damage by restoring blood flow reperfusion in time [3]. Although in situ clot retrieval can improve the recanalization rate of patients with ischemic stroke (mTICI of $2 \mathrm{~b} / 3$ ) to $80-90 \%$, it still exceeds $73 \%$ of patients who are left with functional disability or death (90 days of $m R S \geq 2$ ) [4]. Therefore, it is 
necessary to explore neuroprotective drug to promote neurological function after IS and improve the prognosis of stroke [5].

Traditional Chinese medicine (TCM) has played an important part in maintaining health for thousands of years. In recent years, traditional Chinese drugs have been reported to possess protective effects on the nervous system, which has attracted the attention of researchers worldwide [6]. Danggui Shaoyao San (DSS) is a famous herbal formula composed of the following 6 raw herbs: Paeoniae Radix Alba (PRA), Atractylodes macrocephala Koidz. (AMK), Chuanxiong Rhizoma (CR), Angelicae Sinensis Radix (ASR), Poria $\operatorname{cocos}$ (Schw.) Wolf (PCW), and Alisma orientale (Sam.) Juz. (AOJ), which has been widely used in the treatment of various gynecological diseases [7, 8]. Recently, it was found that DSS is a potential therapeutic agent for the treatment of cognitive impairment and depression [9]. It is reported that DSS not only prevents cognitive impairment from Alzheimer's disease (AD) but also improves microcirculation in patients with asymptomatic cerebral infarction [10]. A number of studies indicate the potentials of DSS for improving neurological functions in poststroke treatment $[11,12]$. DSS treatment also promotes focal angiogenesis and neurogenesis, attenuates neurological deficit scores, and improves memory functions in experimental rat models of cerebral ischemic-reperfusion injury $[13,14]$. However, the underlying neuroprotection mechanisms of DSS against IS remain largely unknown.

Compared with the single-target therapeutic effect of chemical drugs, TCM compound ingredients have the overall therapeutic effect, which is usually modulated through various pathways and targets [15]. System biology, such as network pharmacology, contributes to reveal the biological networks in which drugs work. The integration strategy of network biology and multidirectional pharmacology is conducive to expand the available drug target space and is expected to enhance therapeutic efficacy, elevate clinical trial success rate, and decrease drug discovery costs [16]. In recent years, it has been well applied for drug discovery, especially in the field of research and development of Chinese medicine formulae [17].

According to the description above, DSS is an ideal TCM in the application of treatment to IS. However, the underlying pharmacological mechanisms of DSS on IS treatment remain unclear. In this study, we used the emerging traditional Chinese medicine network pharmacology method to predict the targets of DSS and systematically predict the mechanism of action of DSS in IS. An outline of the method is shown in Figure 1. Our results offered the systematic mechanism of DSS in the treatment of IS and clearly clarified the synergy mechanism of DSS multicomponent and multitarget.

\section{Materials and Methods}

2.1. DSS Ingredient Collection and Target Gene Prediction. The traditional Chinese medicine systems pharmacology database and analysis platform (TCMSP, https://tcmsp-e. $\mathrm{com} /$ ) was used for the active ingredient screening [18]. The names of herbs were used as the keywords to retrieve all components. We filtered active compounds by setting the pharmacokinetic index that the oral bioavailability (OB) was greater than $30 \%$ and the drug-like (DL) index was $>0.18$. Target genes were predicted using TCMSP, the PubChem website (pubchem.ncbi.nlm.nih.gov), and the PharmMapper database (http://www.lilabecust.cn/pharmmapper) after identifying the active ingredients [19].

\subsection{Acquisition of Potential Therapeutic Targets for DSS Anti-IS.} Online Mendelian Inheritance in Man (OMIM, http:// omim.org) database, Comparative Toxicogenomics Database (CTD, http://ctdbase.org/) [20], and Therapeutic Target Database (TTD, http://db.idrblab.net/ttd/) were selected to obtain IS-related target gene [21]. All the databases used "ischemic stroke" as the keyword. The potential therapeutic targets of DSS in the treatment of IS were derived from the overlapping targets of DSS-related targets and IS-related targets. Subsequently, we used Cytoscape software (version 3.7.2) to construct a network diagram of "Drug active ingredient-target gene interaction network." The topology analysis of the composition-target network was carried out using the function of "Network Analyzer" in the software.

2.3. Protein-Protein Interaction (PPI) Analysis. The obtained intersection targets were inputted to the STRING database (http://string-db.org/cgi/inpup.pl) with the species set to "Homo sapiens" to construct a protein-protein interaction (PPI) network [22]. The minimum required interaction score was set to "highest confidence (0.900)," and the hide disconnected nodes were set. Then, we obtained the PPI network. Next, the interaction files were downloaded and imported into Cytoscape software (version 3.7.2) to visualize the PPI network and analyze the topological value [23]. Then, the targets with the degree and betweenness centrality (BC), which were greater than the average value, were recognized as the key targets of DSS for the treatment of IS.

2.4. Enrichment Analysis of Gene Ontology (GO) and Kyoto Encyclopedia of Genes and Genomes (KEGG). The predicted targets were imported into the Database for Annotation, Visualization, and Integrated Discovery (DAVID database, https://david.ncifcrf.gov) for GO and KEGG enrichment analyses [24]. GO enrichment analysis includes biological process (BP), cellular component (CC), and molecular function (MF). The enrichment results of GO and KEGG were obtained by setting the FDR value, and the bubble map was made according to the enrichment results [25].

2.5. Molecular Docking. The top three key compounds analyzed by "Network Analyzer" were obtained in the TCMSP database in mol2 format, and the top three key target proteins in the PPI network were obtained in the PDB database (http://www.rcsb.org). The software AutoDock Vina was used to carry out molecular docking between the key active ingredients and the key targets [26]. The binding energy was used as the evaluation index to evaluate the 


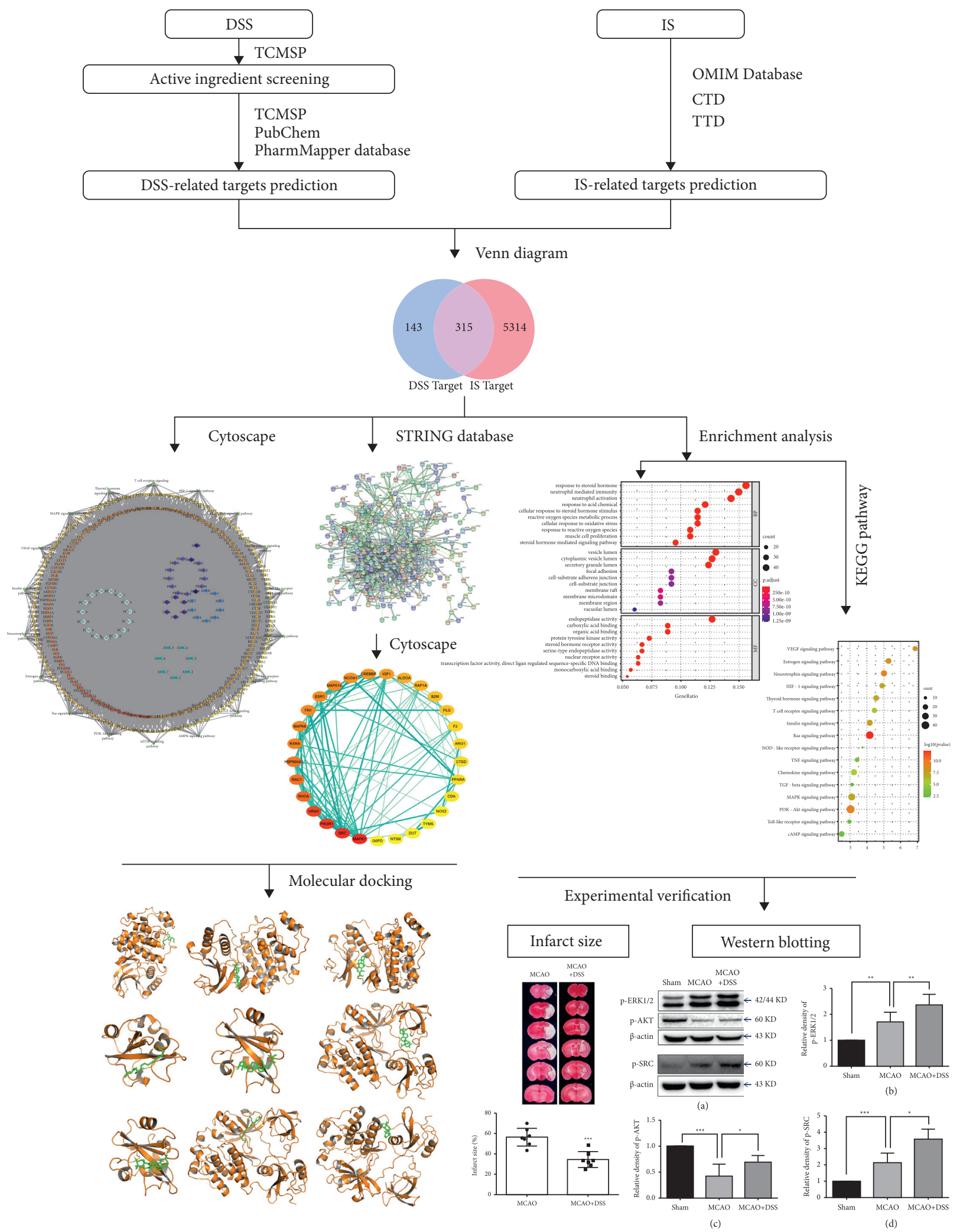

Figure 1: Flowchart of this study. 
docking results, and the results were displayed by the software PyMOL [27].

2.6. Animal Model and Drug Administration. All animal experiments were approved by the Institutional Animal Care and Use Committee of Capital Medical University (XW20210228-1) and in accordance with the principles outlined in the National Institutes of Health Guide for the Care and Use of Laboratory Animals. Male C57/BL6 mice (21-23 g) were used. Transient focal ischemia was induced by right middle cerebral artery occlusion (MCAO) using the intraluminal vascular occlusion method as previously described [28]. In brief, the right common carotid artery and the right external carotid artery (ECA) were exposed. The ECA was then dissected distally, ligated, and coagulated. The right MCA was occluded using a heparinized intraluminal filament. After $60 \mathrm{~min}$, the suture was withdrawn. During surgery, rectal temperature was maintained at $37 \pm 0.5^{\circ} \mathrm{C}$ with a thermostat-controlled heating pad. Sham-operated mice underwent an identical surgery except that the MCA was not occluded. Sixty minutes after occlusion, the filament was removed and a laser speckle contrast imaging (PSI System, Perimed Inc.) was used to observe the local cerebral blood flow. The mice were randomly assigned to the sham, $\mathrm{MCAO}$, and MCAO + DSS groups. The MCAO + DSS group was administered DSS $(20 \mathrm{~g} / \mathrm{kg})$ via the intragastric route at the time of reperfusion.

2.7. Preparation ofDSS. The materials of DSS were purchased from Tong Ren Tang Pharmaceutical Company (Beijing, China) and were then authenticated by Dr. Weipeng Yang in the China Academy of Chinese Medical Sciences. The DSS dilution was prepared as described previously [14]. In brief, the 6 raw herbs were mixed in their dry weight ratios of $3: 16$ : 3:8:4:4 (ASR:PRA:CR:AOJ: PCW:AMK). The mixture was soaked in distilled water $(1: 8 \mathrm{w} / \mathrm{v})$ for 30 minutes at room temperature, boiled for $1.5 \mathrm{~h}$, and the extract was filtered thereafter. The boiling and extraction procedures were repeated three times. The extracted filtrate was concentrated using a rotary evaporator, and the final concentration of the extract is $1 \mathrm{~g} / \mathrm{ml}$ (equivalent to the dry weight of the raw materials). The DSS extract was then stored at $4^{\circ} \mathrm{C}$.

2.8. Two-Dimensional Laser Speckle Imaging. Regional cerebral blood flow (CBF) was monitored by the two-dimensional laser speckle imaging before ischemia and after the onset of ischemia (10 min after MCAO). We calculated the blood flow ratio of the two cerebral hemispheres (right/ left), and at a ratio less than $20 \%$, the MCAO model was considered successful (Figures 2(a)-2(b)).

2.9. Infarct Size Measurement. Infarct size was measured according to previous methods [28]. Twenty-four hours after surgery, the mice ( $n=7$ per group) were anesthetized with $1 \%$ pentobarbital sodium, and then, the brains were removed and sectioned coronally at $1-\mathrm{mm}$ intervals to generate 6 slices. The slices were then incubated with $2 \%$ solution of 2, 3, 4-triphenyltetrazolium chloride (TTC). The infarct area and the corresponding contralateral area were measured by a blinded observer using the Image-Pro Plus software 5.0 (Rockville, MD, USA). Infarct size was calculated as a percentage of the size of contralateral hemisphere.

2.10. Western Blot. Tissue samples were collected from the ischemic hemisphere at 24 hours and 7 days, respectively, after reperfusion for the Western blot analysis. The samples at 24 hours after reperfusion were used for the detection of phosphorylated MAPK1 (p-ERK1/2) and phosphorylated RACalpha serine/threonine-protein kinase (p-AKT). The samples at 7 days after reperfusion were used for the detection of phosphorylated proto-oncogene tyrosine-protein kinase Src (pSRC). Protein $(40 \mu \mathrm{g})$ was electrophoresed on $10 \%$ SDS polyacrylamide gels (Beijing Biotides Biotechnology Co., Ltd, Beijing, China) and then transferred to a polyvinylidene fluoride membrane (Millipore Corporation, USA). The membrane was probed with primary antibody: anti-p-ERK1/2 antibody (Cell Signaling; $1: 1000$ dilution), anti-p-AKT antibody (Cell Signaling; 1:1000 dilution), and anti-p-SRC antibody (Cell Signaling; 1:1000 dilution). The specific reaction was visualized through the use of a chemiluminescent substrate (GE Healthcare, UK) [29]. $\beta$-Actin was used to verify equal loading. The optical density of protein was measured using ImageJ software (NIH, Bethesda, MD, USA) according to the manufacturer's instructions ( $n=7$ per group).

2.11. Statistical Analysis. For two groups, the differences were analyzed for statistical significance by Student's $t$-test. For three or more groups, the differences were analyzed for statistical significance by the one-way ANOVA followed by Tukey's post hoc test. All the data were expressed as mean \pm SD. The statistical analysis was performed with SPSS for Windows, version 21.0 (SPSS Inc.). The $p$-value $<0.05$ was considered significant.

\section{Results}

3.1. DSS Ingredient Collection and Target Gene Prediction. According to the two screening conditions of $\mathrm{OB}$ value and DL index, 54 active ingredients of DSS were obtained from TCMSP, including 13 ingredients in PRA, 7 ingredients in $\mathrm{CR}, 7$ ingredients in AMK, 2 ingredients in ASR, 15 ingredients in PCW, and 10 ingredients in AOJ. ASR and PRA share one ingredient. PRA, AOJ, and CR share one ingredient (Table 1). Then, the target genes of 54 active ingredients were collected for target gene prediction in TCMSP and PubChem. A total of 14978 predicted targets were obtained. After removing the other species gene targets and duplicate values, 458 relevant human gene targets were obtained, including ASR (232), PRA (432), AOJ (377), CR(433), PCW (409), and AMK (359).

\subsection{Drug Active Ingredient-Target Gene Interaction Network.} The overlapping targets of DSS-related targets and IS-related targets were considered as potential therapeutic targets for 

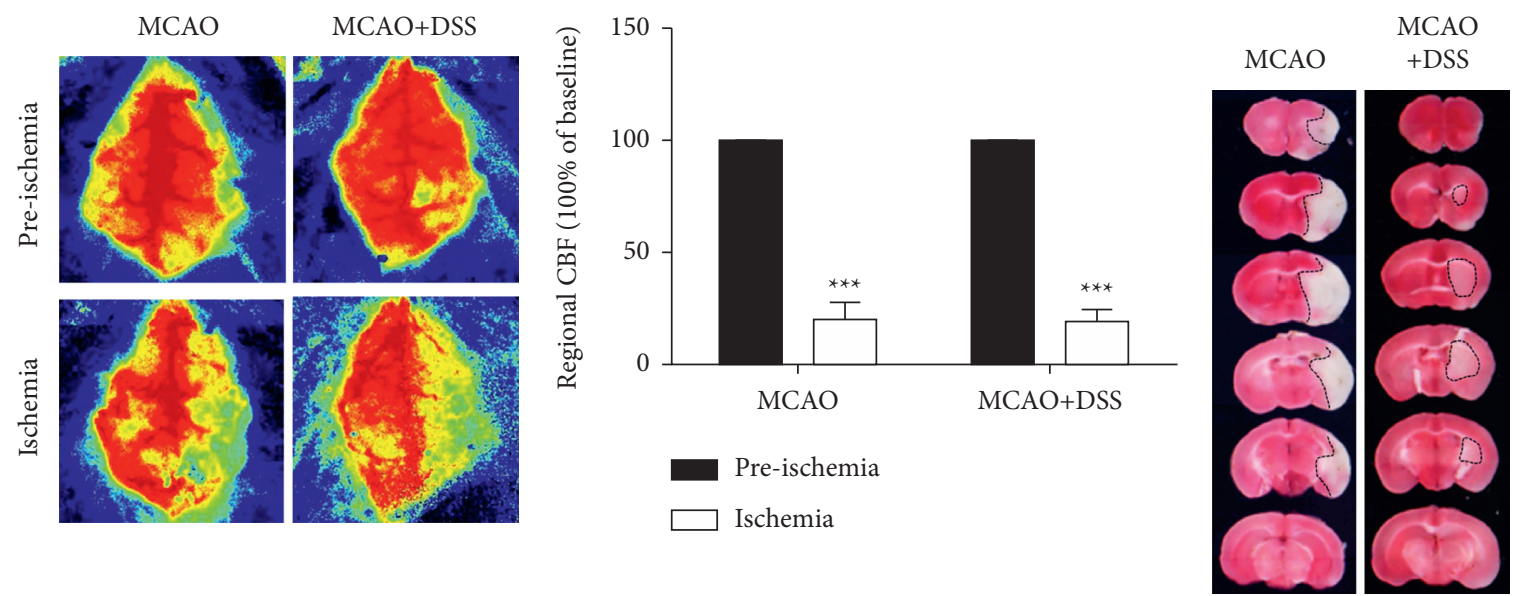

(a)

(b)

(c)

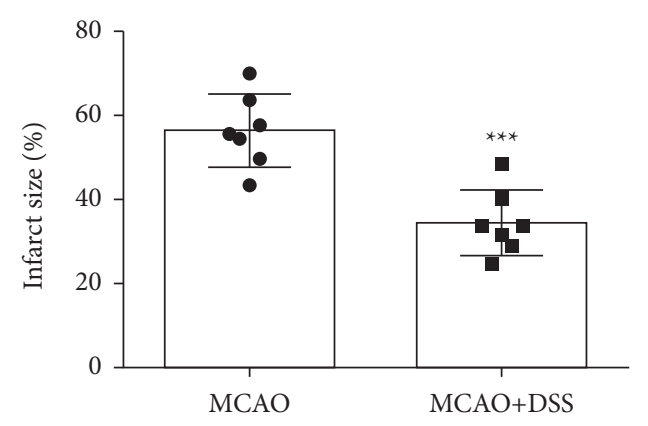

(d)

FIGURE 2: DSS treatment reduced infarct volume. (a) Regional cerebral blood flow (CBF) was monitored by the two-dimensional laser speckle imaging. (b) The blood flow ratio of two cerebral hemispheres (right/left), and a ratio less than $20 \%$ considered that the MCAO model is successful $(P<0.05)$. (c) Representative brain slices with infarcts stained by triphenyltetrazolium chloride from each group at $24 \mathrm{~h}$ after MCAO. (d) Quantification of infarct size at $24 \mathrm{~h}$ after MCAO. $N=7$ per group. ${ }^{* * *} P<0.001$, vs. MCAO group, by Student's $t$-test.

DSS anti-IS. As shown in Figure 1, a total of 315 intersection targets were obtained by screening drug ingredient targets and IS targets (Figure 3(a)). Six kinds of drugs have 182 common targets (Table 2) (Figure 3(b)). Drug active component-target gene interaction network contained 366 nodes (including 51 herb compound nodes and 315 target gene nodes) and 11146 edges. The degree value of a node represents the number of lines connected to the node in the network. The larger node means more importance. Among 315 target gene nodes, the higher the degree of disease correlation, the redder the color (Figure 4). In addition, in the network pharmacological map of a single herb, the higher the degree value of the target gene to the herb, the closer it is to the herb component (Figure 5). The topology analysis showed that the average degree value of each node in the network was 208.4706 , and the average medium was 0.016732 . There were 17 compound nodes with both degree and intermediate values above the mean (Table 3 ), suggesting that these compounds may be key compounds in the treatment of stroke.

3.3. Construction of the PPI Network and Core Target Screening. 315 selected target genes were introduced into the STRING database to construct PPI network
(Figure 6(a)). The PPI network contains 268 nodes and 1184 edges, indicating that 268 targets can interact with each other in the network, resulting in a total of 1184 interactions. After the analysis of topology parameters, the average node degree is 8.670412, and the average medium is 0.20796 . There are 30 nodes with degree and intermediate values above the average, which were considered to be core targets of DSS in the treatment of IS (Figure 6(b), Table 4). According to the degree values, the top 10 core targets were identified as MAPK1, SRC, PIK3R1, HRAS, AKT1, RHOA, RAC1, HSP90AA1, RXRA, and FN1.

3.4. GO and KEGG Enrichment Analyses. 315 targets screened by the DAVID database were used for GO functional enrichment analysis and KEGG signaling pathway enrichment analysis (FDR $<0.01)$. As a result, we obtained 417 GO terms, including 288 biological processes (BPs), 86 molecular functions (MFs), and 43 cellular components (CCs). BP mainly includes negative regulation of apoptosis, steroid hormone-mediated signaling pathway, neutrophil activation, and cellular response to oxidative stress. MF mainly includes the activity of steroid hormone receptor and protein tyrosine kinase. CC mainly includes cytoplasm and extracellular bodies. The top 10 screened BP, MF, and CC 
TABLE 1: Pharmaceutical ingredients.

\begin{tabular}{|c|c|c|c|c|}
\hline $\begin{array}{l}\text { Chinese } \\
\text { name }\end{array}$ & Latin name & Molecule ID & Active ingredients & $\begin{array}{l}\text { Ingredient } \\
\text { code }\end{array}$ \\
\hline \multirow{2}{*}{ Danggui } & \multirow{2}{*}{ Angelicae Sinensis Radix } & MOL000358 & Beta-sitosterol & ASR-1 \\
\hline & & MOL000449 & Stigmasterol & ASR-2 \\
\hline \multirow{13}{*}{ Baishao } & \multirow{13}{*}{ Paeoniae Radix Alba } & MOL001921 & Lactiflorin & PRA-1 \\
\hline & & MOL001924 & Paeoniflorin & PRA-2 \\
\hline & & MOL000211 & Mairin & PRA-3 \\
\hline & & MOL000358 & Beta-sitosterol & ASR-1 \\
\hline & & MOL000359 & Sitosterol & PRA-4 \\
\hline & & MOL001930 & Benzoyl paeoniflorin & PRA-5 \\
\hline & & MOL001919 & Palbinone & PRA-6 \\
\hline & & MOL001925 & Paeoniflorin & PRA-7 \\
\hline & & MOL001910 & $\begin{array}{l}\text { 11alpha,12alpha-epoxy-3beta-23-Dihydroxy-30-nor--olean-20- } \\
\text { en-28,12beta-olide }\end{array}$ & PRA-8 \\
\hline & & MOL001918 & Paeoniflorin genome & PRA-9 \\
\hline & & MOL001928 & Albiflorin & PRA-10 \\
\hline & & MOL000492 & Cianidanol & PRA-11 \\
\hline & & MOL000422 & Kaempferol & PRA-12 \\
\hline \multirow{10}{*}{ Zexie } & \multirow{10}{*}{$\begin{array}{c}\text { Alisma Orientale (Sam.) } \\
\text { Juz. }\end{array}$} & MOL000856 & Alisol C monoacetate & AOJ-1 \\
\hline & & MOL000853 & Alisol B & AOJ -2 \\
\hline & & MOL000832 & Alisol B 23-acetate & AOJ-3 \\
\hline & & MOL000830 & Alisol B & AOJ -4 \\
\hline & & MOL000854 & Alisol C & AOJ-5 \\
\hline & & MOL000862 & Alisol B 23-acetate & AOJ-6 \\
\hline & & MOL000831 & Alisol B monoacetate & AOJ-7 \\
\hline & & MOL000849 & $16 \beta$-Methoxyalisol B monoacetate & AOJ-8 \\
\hline & & MOL000359 & Sitosterol & PRA-4 \\
\hline & & MOL002464 & 1-Monolinolein & AOJ-9 \\
\hline \multirow{7}{*}{ Chuanxiong } & \multirow{7}{*}{ Chuanxiong Rhizoma } & MOL000359 & Sitosterol & PRA-4 \\
\hline & & MOL002157 & Wallichilide & CR-1 \\
\hline & & MOL000433 & Folsaeure & CR-2 \\
\hline & & MOL002135 & Myricanone & CR-3 \\
\hline & & MOL002140 & Perlolyrine & CR-4 \\
\hline & & MOL002151 & Senkyunone & CR-5 \\
\hline & & MOL001494 & Mandenol & CR-6 \\
\hline \multirow{15}{*}{ Fuling } & \multirow{15}{*}{ Poria $\operatorname{cocos}$ (Schw.) Wolf. } & MOL000300 & Dehydroeburicoic acid & PCW-1 \\
\hline & & MOL000285 & Polyporenic acid C & PCW-2 \\
\hline & & MOL000280 & Dehydrotumulosic acid & PCW -3 \\
\hline & & MOL000273 & 16alpha-Hydroxydehydrotrametenolic acid & PCW-4 \\
\hline & & MOL000283 & Ergosterol peroxide & PCW-5 \\
\hline & & MOL000276 & 7, 9 (11)-dehydropachymic acid & PCW-6 \\
\hline & & MOL000289 & Pachymic acid & PCW-7 \\
\hline & & MOL000287 & Eburicoic acid & PCW-8 \\
\hline & & MOL000275 & Trametenolic acid & PCW-9 \\
\hline & & MOL000279 & Cerevisterol & PCW-10 \\
\hline & & MOL000290 & Poricoic acid A & PCW-11 \\
\hline & & MOL000296 & Hederagenin & PCW-12 \\
\hline & & MOL000292 & Poricoic acid C & PCW-13 \\
\hline & & MOL000291 & Poricoic acid B & PCW-14 \\
\hline & & MOL000282 & Stellasterol & PCW-15 \\
\hline \multirow{7}{*}{ Baizhu } & \multirow{7}{*}{$\begin{array}{l}\text { Atractylodes macrocephala } \\
\text { Koidz. }\end{array}$} & MOL000033 & (24S)-24-Propylcholesta-5-ene-3beta-ol & AMK-1 \\
\hline & & MOL000028 & A-Amyrin & AMK-2 \\
\hline & & MOL000021 & 14-Acetyl-12-senecioyl-2E, 8E, 10E-atractylentriol & AMK-3 \\
\hline & & MOL000022 & 14-Acetyl-12-senecioyl-2E, 8Z, 10E-atractylentriol & AMK-4 \\
\hline & & MOL000020 & 12-Senecioyl-2E, $8 \mathrm{E}, 10 \mathrm{E}$-atractylentriol & AMK-5 \\
\hline & & MOL000049 & $3 \beta$-Acetoxyatractylone & AMK-6 \\
\hline & & MOL000072 & $8 \beta$-Ethoxy atractylenolide III & AMK-7 \\
\hline
\end{tabular}



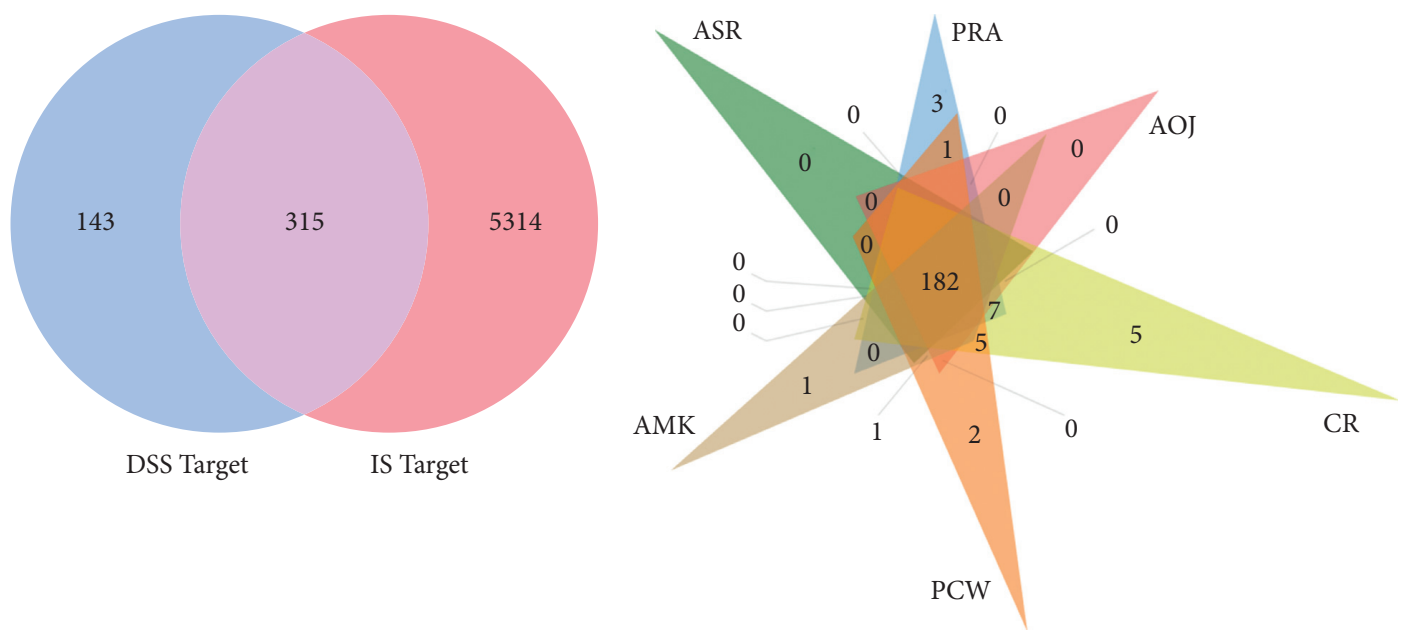

(a)

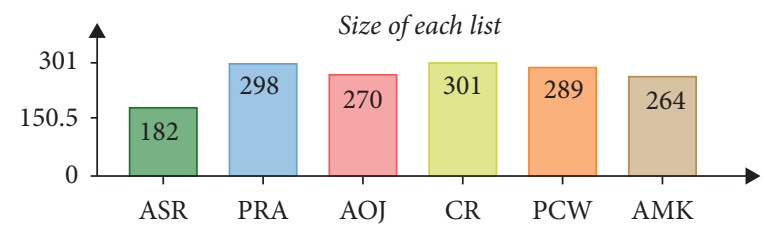

(b)

Figure 3: Identification of the drug-target interaction. (a) Venn diagram. (b) Drug-disease target analysis of traditional Chinese medicine. 182 represents the common targets of six Chinese medicines, and other figures indicate the unique targets of each Chinese medicine. PRA, Paeoniae Radix Alba. AMK, Atractylodes macrocephala Koidz., CR, Chuanxiong Rhizoma. ASR, Angelicae Sinensis Radix. PCW, Poria cocos (Schw.) Wolf. AOJ, Alisma orientale (Sam.) Juz.

TABLE 2: Drug active ingredient-target gene (common targets of six drugs).

\begin{tabular}{|c|c|}
\hline Gene & Gene ID \\
\hline CASP3 & 836 \\
\hline SOD2 & 6648 \\
\hline NOS3 & 4846 \\
\hline HMOX1 & 3162 \\
\hline MAPK1 & 5594 \\
\hline $\mathrm{F} 2$ & 2147 \\
\hline MMP2 & 4313 \\
\hline GSK3B & 2932 \\
\hline МAPК8 & 5599 \\
\hline IL-2 & 3558 \\
\hline PPARG & 5468 \\
\hline NR3C1 & 2908 \\
\hline MAPK14 & 1432 \\
\hline REN & 5972 \\
\hline F3 & 2152 \\
\hline IGF-1 & 3479 \\
\hline BCL2L1 & 598 \\
\hline PARP1 & 142 \\
\hline EGFR & 1956 \\
\hline CASP7 & 840 \\
\hline $\mathrm{ALB}$ & 213 \\
\hline CYP19A1 & 1588 \\
\hline PPARA & 5465 \\
\hline GSR & 2936 \\
\hline FABP4 & 2167 \\
\hline IGF-1R & 3480 \\
\hline MMP3 & 4314 \\
\hline MAOA & 4128 \\
\hline
\end{tabular}


TABle 2: Continued.

\begin{tabular}{|c|c|}
\hline Gene & Gene ID \\
\hline NQO1 & 1728 \\
\hline MMP13 & 4322 \\
\hline HSP90AA1 & 3320 \\
\hline CCNA2 & 890 \\
\hline AKR1C3 & 8644 \\
\hline MDM2 & 4193 \\
\hline TGFBR1 & 7046 \\
\hline CALM1 & 801 \\
\hline PGR & 5241 \\
\hline TGFB2 & 7042 \\
\hline CYP2C9 & 1559 \\
\hline ESR1 & 2099 \\
\hline NR3C2 & 4306 \\
\hline KDR & 3791 \\
\hline GSTP1 & 2950 \\
\hline F7 & 2155 \\
\hline $\mathrm{AR}$ & 367 \\
\hline NR1I2 & 8856 \\
\hline AURKA & 6790 \\
\hline MAP2K1 & 5604 \\
\hline $\mathrm{B} 2 \mathrm{M}$ & 567 \\
\hline CASP1 & 834 \\
\hline HSPA 8 & 3312 \\
\hline MAOB & 4129 \\
\hline FGFR1 & 2260 \\
\hline HSP90AB1 & 3326 \\
\hline HMGCR & 3156 \\
\hline JAK2 & 3717 \\
\hline MME & 4311 \\
\hline CTSB & 1508 \\
\hline LCN2 & 3934 \\
\hline HSD11B1 & 3290 \\
\hline SRC & 6714 \\
\hline XIAP & 331 \\
\hline SULT1E1 & 6783 \\
\hline VDR & 7421 \\
\hline GSTA1 & 2938 \\
\hline GCK & 2645 \\
\hline INSR & 3643 \\
\hline CDK2 & 1017 \\
\hline NCOA2 & 10499 \\
\hline S100A9 & 6280 \\
\hline FABP5 & 2171 \\
\hline BMP2 & 650 \\
\hline TEK & 7010 \\
\hline MET & 4233 \\
\hline CDK6 & 1021 \\
\hline CYP2C8 & 1558 \\
\hline CTSK & 1513 \\
\hline PSAP & 5660 \\
\hline THRB & 7068 \\
\hline PDPK1 & 5170 \\
\hline ADAM17 & 6868 \\
\hline KIT & 3815 \\
\hline EPHX2 & 2053 \\
\hline FKBP1A & 2280 \\
\hline MMP12 & 4321 \\
\hline CTSS & 1520 \\
\hline MAPK10 & 5602 \\
\hline FABP3 & 2170 \\
\hline
\end{tabular}


TABle 2: Continued.

\begin{tabular}{|c|c|}
\hline Gene & Gene ID \\
\hline RARA & 5914 \\
\hline MAPKAPK2 & 9261 \\
\hline PIK3R1 & 5295 \\
\hline PDE4D & 5144 \\
\hline LSS & 4047 \\
\hline AKT2 & 208 \\
\hline PIM1 & 5292 \\
\hline AKR1B1 & 231 \\
\hline PLA2G2A & 5320 \\
\hline NR1I3 & 9970 \\
\hline OAT & 4942 \\
\hline DUSP6 & 1848 \\
\hline PGF & 5228 \\
\hline CHEK1 & 1111 \\
\hline ESRRA & 2101 \\
\hline PPIA & 5478 \\
\hline TNNC1 & 7134 \\
\hline AKR1C1 & 1645 \\
\hline GSTM1 & 2944 \\
\hline $\mathrm{HCK}$ & 3055 \\
\hline SULT2A1 & 6822 \\
\hline PDK2 & 5164 \\
\hline F10 & 2159 \\
\hline RORA & 6095 \\
\hline NR1H3 & 10062 \\
\hline AKR1C2 & 1646 \\
\hline EIF4E & 1977 \\
\hline BRAF & 673 \\
\hline $\mathrm{CA} 2$ & 760 \\
\hline AMD1 & 262 \\
\hline MMP8 & 4317 \\
\hline JAK3 & 3718 \\
\hline NCOA1 & 8648 \\
\hline NR1H4 & 9971 \\
\hline THRA & 7067 \\
\hline PDE4B & 5142 \\
\hline PTPN11 & 5781 \\
\hline GART & 2618 \\
\hline RBP4 & 5950 \\
\hline PDE5A & 8654 \\
\hline BACE1 & 23621 \\
\hline WAS & 7454 \\
\hline GRB2 & 2885 \\
\hline PTPN1 & 5770 \\
\hline PROCR & 10544 \\
\hline SDS & 10993 \\
\hline PPP5C & 5536 \\
\hline PRKCQ & 5588 \\
\hline $\mathrm{BCHE}$ & 590 \\
\hline ACADM & 34 \\
\hline DHFR & 1719 \\
\hline FGFR2 & 2263 \\
\hline TTR & 7276 \\
\hline PYGL & 5836 \\
\hline GC & 2638 \\
\hline ANXA5 & 308 \\
\hline CES1 & 1066 \\
\hline LCK & 3932 \\
\hline DPP4 & 1803 \\
\hline FKBP1B & 2281 \\
\hline
\end{tabular}


TABLE 2: Continued.

\begin{tabular}{|c|c|}
\hline Gene & Gene ID \\
\hline FABP7 & 2173 \\
\hline $\mathrm{FECH}$ & 2235 \\
\hline STS & 412 \\
\hline ELANE & 1991 \\
\hline BAG1 & 573 \\
\hline ERBB4 & 2066 \\
\hline GM2A & 2760 \\
\hline TPX2 & 22974 \\
\hline PLK1 & 5347 \\
\hline TYMS & 7298 \\
\hline DCK & 1633 \\
\hline BHMT & 635 \\
\hline CTNNA1 & 1495 \\
\hline CRABP2 & 1382 \\
\hline ESRRG & 2104 \\
\hline RXRA & 6256 \\
\hline MTAP & 4507 \\
\hline SHBG & 6462 \\
\hline NCOA5 & 57727 \\
\hline PPARD & 5467 \\
\hline ABL1 & 25 \\
\hline TTPA & 7274 \\
\hline FKBP3 & 2287 \\
\hline GRB14 & 2888 \\
\hline РСТР & 58488 \\
\hline CTSF & 8722 \\
\hline $\mathrm{ADK}$ & 132 \\
\hline HSD17B1 & 3292 \\
\hline KIF11 & 3832 \\
\hline RFK & 55312 \\
\hline FNTB & 2342 \\
\hline YARS1 & 8565 \\
\hline HNMT & 3176 \\
\hline BCAT2 & 587 \\
\hline
\end{tabular}

were selected as bubble charts through $\mathrm{R}$ language-related procedures (Figure 7).

The enrichment and screening of KEGG pathways resulted in 119 signaling pathways $(F D R<0.01)$ and 16 pathways related to stroke (Figure 8 ). The main pathway included VEGF signaling pathway, estrogen signaling pathway, neurotrophin signaling pathway, HIF-1 signaling pathway, and thyroid hormone signaling pathway.

3.5. Molecular Docking. The top 3 key target proteins (MAPK1, PIK3R1, and SRC) in PPI network were selected to dock with the top 3 key active compounds ("11alpha, 12alpha-epoxy-3beta-23-dihydroxy-30-norolean-20-en-28,12beta-olide," "eburicoic acid," "12senecioyl-2E, 8E, 10E-atractylentriol”). The PyMOL software was used to visualize the docking results of key active ingredients and core targets, as shown in Figure 9 and Table 5.

3.6. DSS Treatment Reduced Infarct Size. To confirm the neuroprotection of DSS in focal ischemic stroke, the MCAO mice were treated with DSS, and 24 hours later, the infarct size was detected by TTC stain. Compared with the MCAO group (56.47 \pm 3.30$)$, the infarct size was reduced by $39 \%$ in the MCAO + DSS group $(34.49 \pm 2.94)$ $(P=0.0003)$ (Figure 2).

3.7. DSS Modulated the Expression of $p-A K T, p-E R K 1 / 2$, and $p$-SRC. To evaluate the performance of the core target screening approach used in this study, we selected the three major target proteins for analysis by the Western blot. The level of p-ERK1/2 was significantly increased in the MCAO mice (MCAO vs. sham, $p<0.01$ ) and further significantly increased after LRIC treatment $(\mathrm{MCAO}+\mathrm{DSS}$ vs. MCAO, $p<0.01$ ) (Figure 10). Moreover, compared with the sham group, the expression of p-AKT in the MCAO group was decreased $(P<0.001)$, but that in the DSS group was significantly upregulated compared with the MCAO group $(p<0.05)$ (Figure 2$)$. We also measured the expression levels of $\mathrm{p}-\mathrm{SRC}$ at day 7 after MCAO surgery, finding that, when compared with the sham group, the levels of $\mathrm{p}$-SRC in the MCAO mice were significantly increased $(P<0.001)$. DSS treatment for 7 days increased the levels of p-SRC compared with those found in the MCAO group $(P<0.05)$ (Figure 10). 


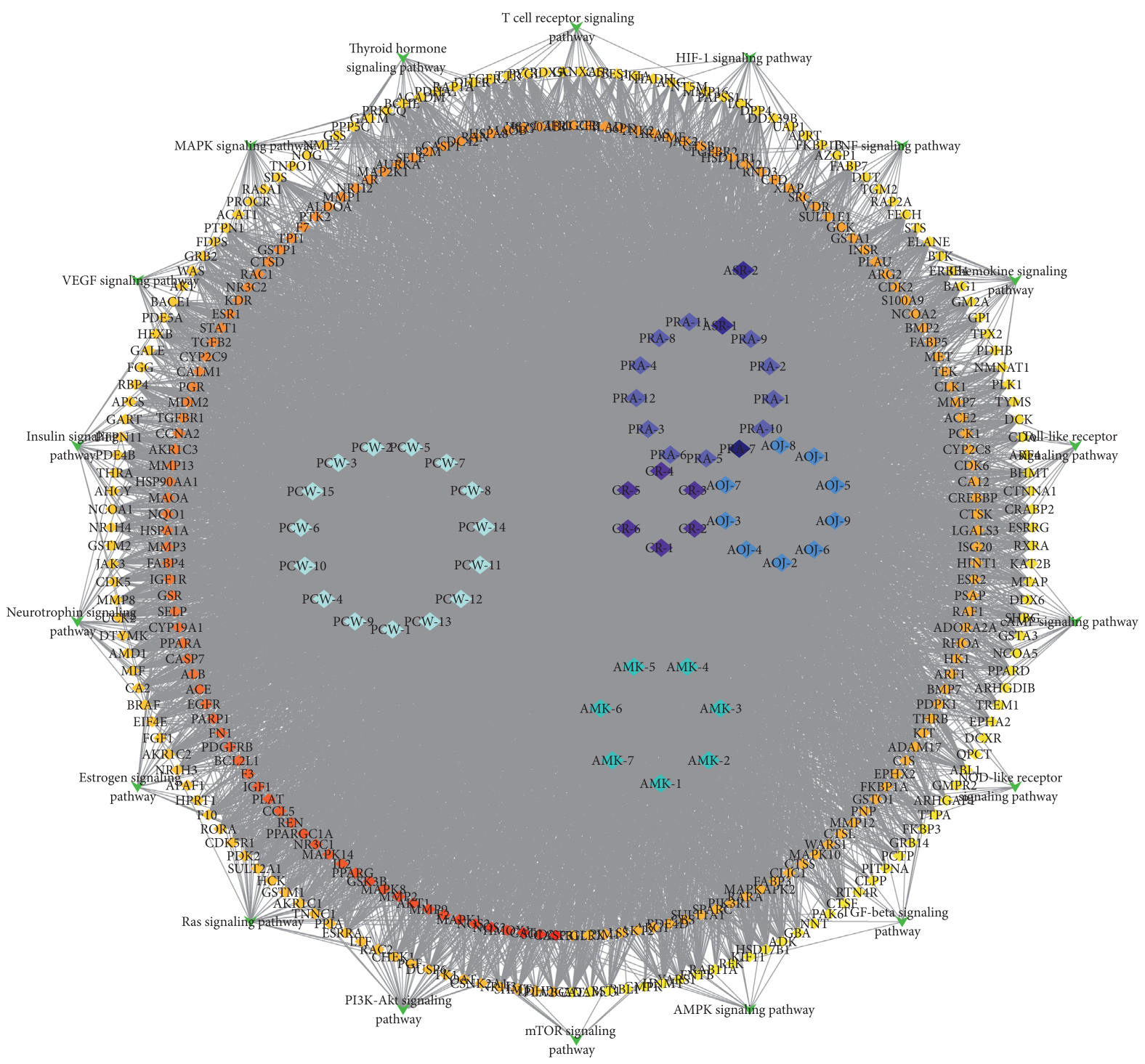

FIGURE 4: DSS prescription compositions of active ingredient-target pathway network. Rhombus represents the active ingredients of herbs contained in DSS. The degree value of a node represents the number of lines connected to the node in the network. The higher the degree value, the more important the node. The round nodes represent disease targets. The higher the degree of disease correlation, the redder the color is.

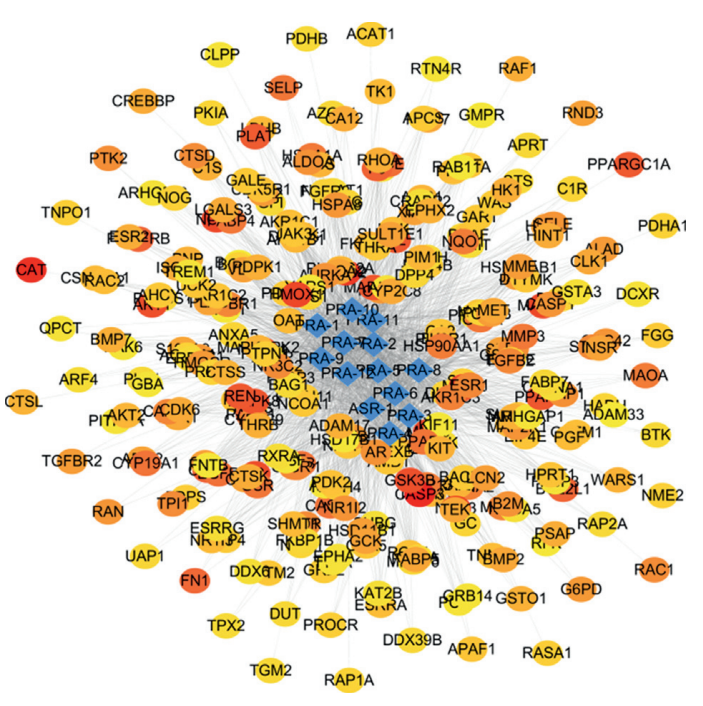

(a)

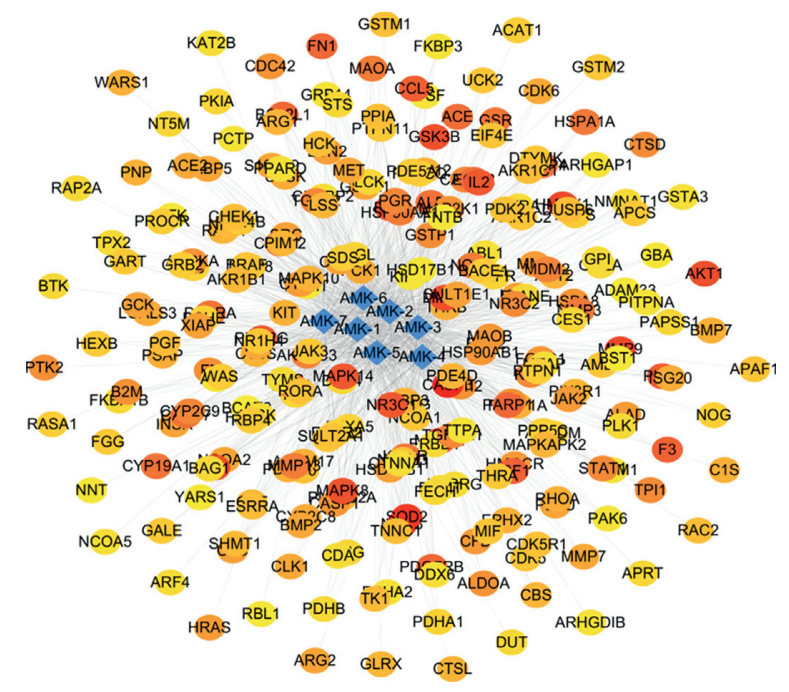

(b)

FIGURE 5: Continued. 


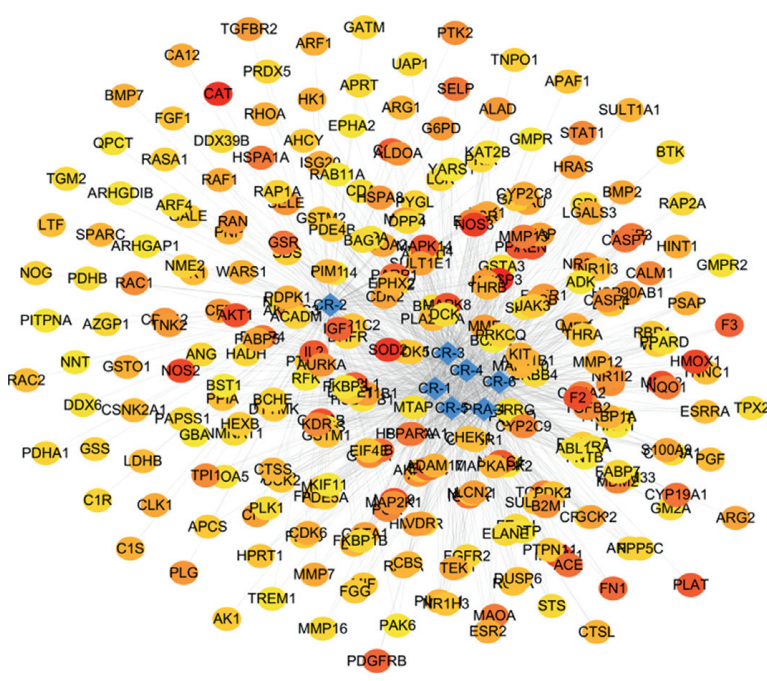

(c)

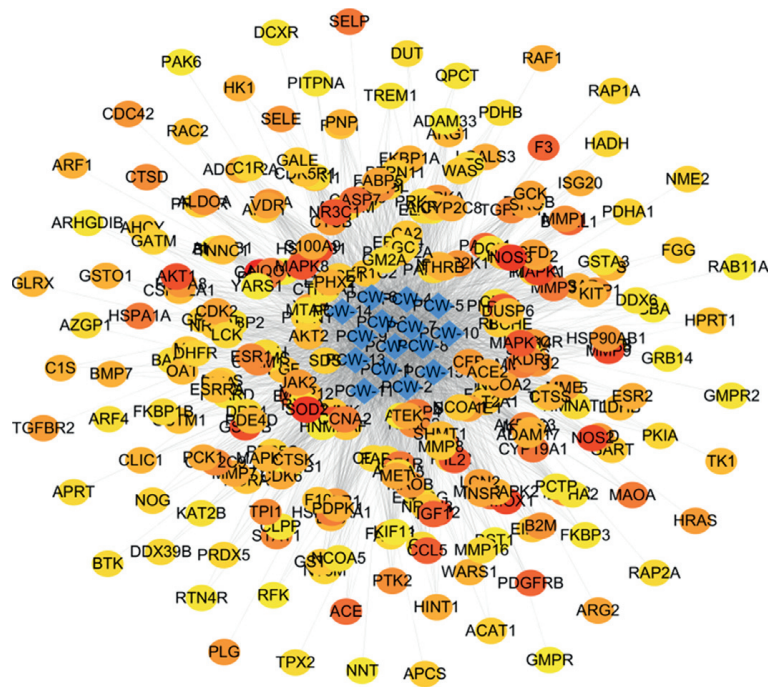

(e)

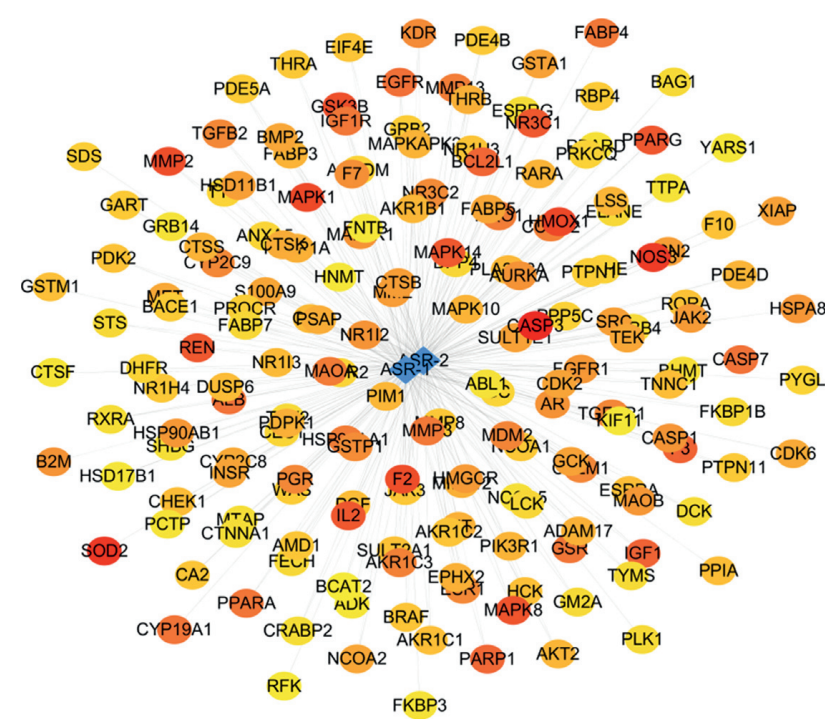

(d)

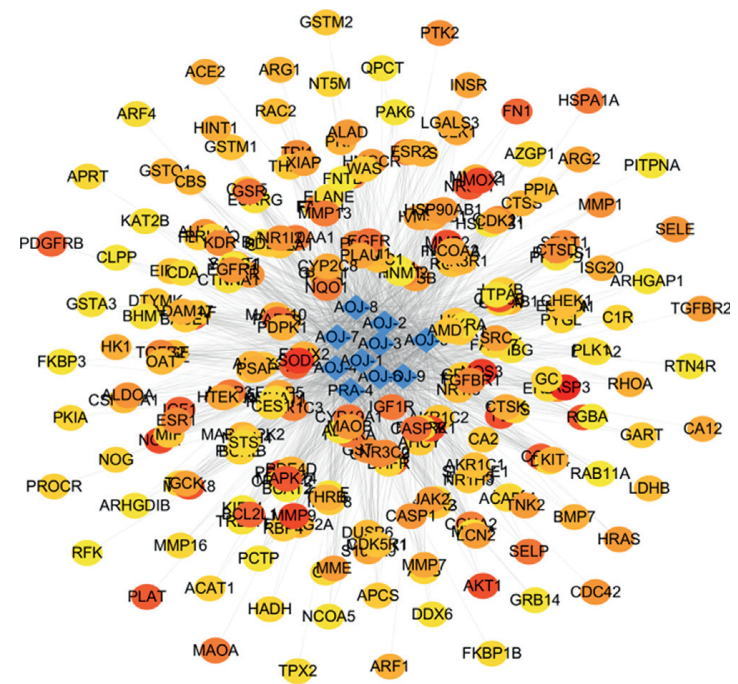

(f)

FIgure 5: The network pharmacological diagram of a single herb. (a) PRA, Paeoniae Radix Alba. (b) AMK, Atractylodes macrocephala Koidz. (c) CR, Chuanxiong Rhizoma. (d) ASR, Angelicae Sinensis Radix. (e) PCW, Poria cocos (Schw.) Wolf. (f) AOJ, Alisma orientale (Sam.) Juz. Rhombus represents the active ingredients of herbs contained in DSS. The round nodes represent disease targets. The higher the degree of disease correlation, the redder the color is. The higher the degree value of the target gene to the herb, the closer it is to the herb component.

TABLE 3: Key compounds. (The average degree value is 208.4706 , and the average medium is 0.016732 .)

\begin{tabular}{|c|c|c|c|}
\hline Compound & Compound ID & Degree & Betweenness centrality \\
\hline 11alpha,12alpha-epoxy-3beta-23-Dihydroxy-30-nor-olean-20-en-28,12beta-olide & PRA-8 & 228 & 0.024789 \\
\hline Eburicoic acid & PCW -8 & 226 & 0.017975 \\
\hline 12-Senecioyl-2E, 8E, 10E-atractylentriol & AMK-5 & 225 & 0.021314 \\
\hline Dehydroeburicoic acid & PCW-1 & 225 & 0.01739 \\
\hline 14-Acetyl-12-senecioyl-2E, 8Z, 10E-atractylentriol & PRA-11 & 223 & 0.028611 \\
\hline Cianidanol & AMK-4 & 223 & 0.01698 \\
\hline Alisol C & AOJ-5 & 221 & 0.017782 \\
\hline 16alpha-Hydroxydehydrotrametenolic acid & PCW-4 & 221 & 0.017585 \\
\hline Poricoic acid C & PCW-13 & 220 & 0.021088 \\
\hline Palbinone & PRA-6 & 219 & 0.024345 \\
\hline Cerevisterol & PCW-10 & 217 & 0.017087 \\
\hline Poricoic acid A & PCW-11 & 216 & 0.02044 \\
\hline Kaempferol & PRA-12 & 215 & 0.032249 \\
\hline Paeoniflorin & PRA-2 & 215 & 0.017069 \\
\hline Lactiflorin & PRA-1 & 214 & 0.016884 \\
\hline Poricoic acid B & PCW-14 & 213 & 0.021272 \\
\hline Myricanone & CR-3 & 209 & 0.041457 \\
\hline
\end{tabular}




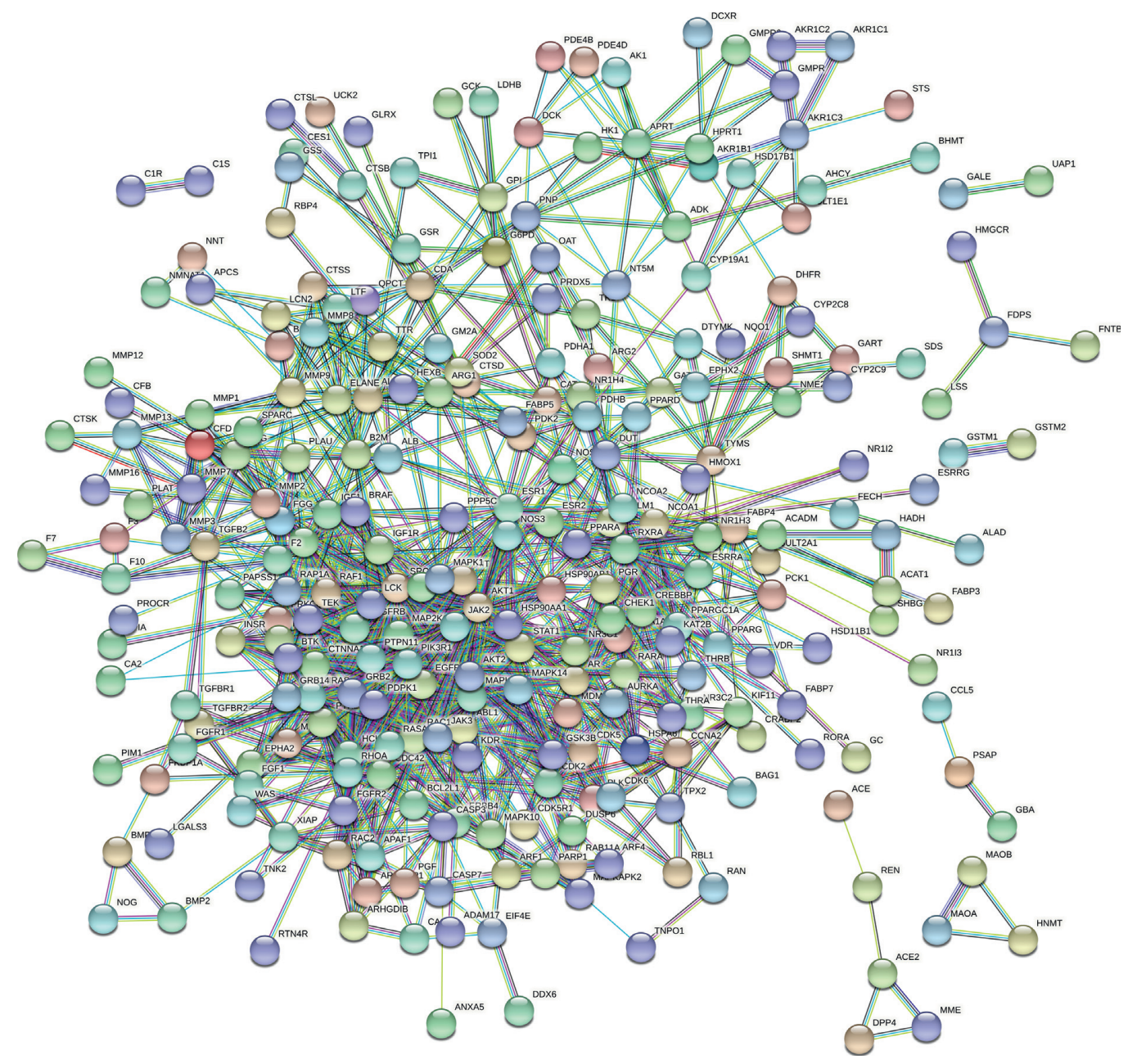

(a)

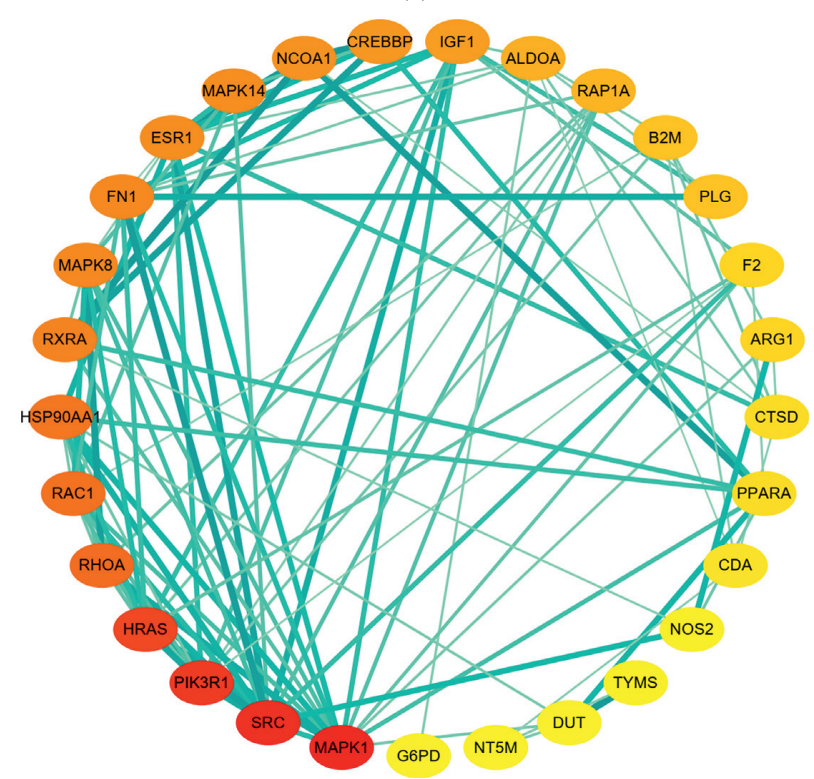

(b)

Figure 6: PPI network and top 30 hub genes for DSS anti-IS. (a) PPI network constructed with the STRING database. (b) Top 30 hub genes for DSS anti-IS were obtained using the degree and betweenness centrality algorithm. The node size and color indicate the degree value. The heavier the color, the greater the degree value and the wider the line and the heavier its color, indicating the closer the connection between the two proteins. 
TABLE 4: Drug targets of IS. (The average degree value is 8.670412 , and the average medium is 0.20796.)

\begin{tabular}{|c|c|c|c|}
\hline Gene & Protein name & Degree & Betweenness centrality \\
\hline MAPK1 & Mitogen-activated protein kinase 1 & 53 & 0.126385 \\
\hline SRC & Proto-oncogene tyrosine-protein kinase Src & 50 & 0.052449 \\
\hline PIK3R1 & & 47 & 0.047873 \\
\hline HRAS & GTPase HRas & 44 & 0.033552 \\
\hline AKT1 & Phosphatidylinositol 3-kinase regulatory subunit alpha & 40 & 0.109791 \\
\hline RHOA & Transforming protein RhoA & 35 & 0.047081 \\
\hline $\mathrm{RAC1}$ & Ras-related C3 botulinum toxin substrate 1 & 34 & 0.0268 \\
\hline HSP90AA1 & Heat shock protein HSP 90-alpha & 33 & 0.059285 \\
\hline RXRA & Retinoic acid receptor alpha, RAR-alpha & 30 & 0.067159 \\
\hline FN1 & Fibronectin 1 & 29 & 0.023024 \\
\hline MAPK8 & Mitogen-activated protein kinase 8 & 29 & 0.022235 \\
\hline ESR1 & Estrogen receptor & 28 & 0.091184 \\
\hline MAPK14 & Mitogen-activated protein kinase 14 & 28 & 0.058202 \\
\hline NCOA1 & Nuclear receptor coactivator 1 & 27 & 0.039326 \\
\hline CREBBP & CREB binding protein & 26 & 0.03777 \\
\hline IGF-1 & Insulin-like growth factor I & 25 & 0.024056 \\
\hline ALDOA & Fructose-bisphosphate aldolase A & 22 & 0.065989 \\
\hline RAP1A & Ras-related protein Rap-1A & 21 & 0.026304 \\
\hline $\mathrm{B} 2 \mathrm{M}$ & Beta-2-microglobulin & 19 & 0.034377 \\
\hline PLG & Plasminogen & 19 & 0.023626 \\
\hline $\mathrm{F} 2$ & Prothrombin & 16 & 0.028418 \\
\hline ARG1 & Arginase-1 & 16 & 0.021575 \\
\hline CTSD & Cathepsin D & 15 & 0.037545 \\
\hline PPARA & Peroxisome proliferator-activated receptor alpha & 15 & 0.028961 \\
\hline CDA & Cytidine deaminase & 14 & 0.059555 \\
\hline TYMS & Thymidylate synthase & 11 & 0.037145 \\
\hline NOS3 & Nitric oxide synthase 3 & 11 & 0.036816 \\
\hline DUT & Deoxyuridine triphosphatase & 9 & 0.057749 \\
\hline NT5M & $5^{\prime}\left(3^{\prime}\right)$-Deoxyribonucleotidase, mitochondrial & 9 & 0.048718 \\
\hline G6PD & Glucose-6-phosphate dehydrogenase & 9 & 0.027099 \\
\hline
\end{tabular}

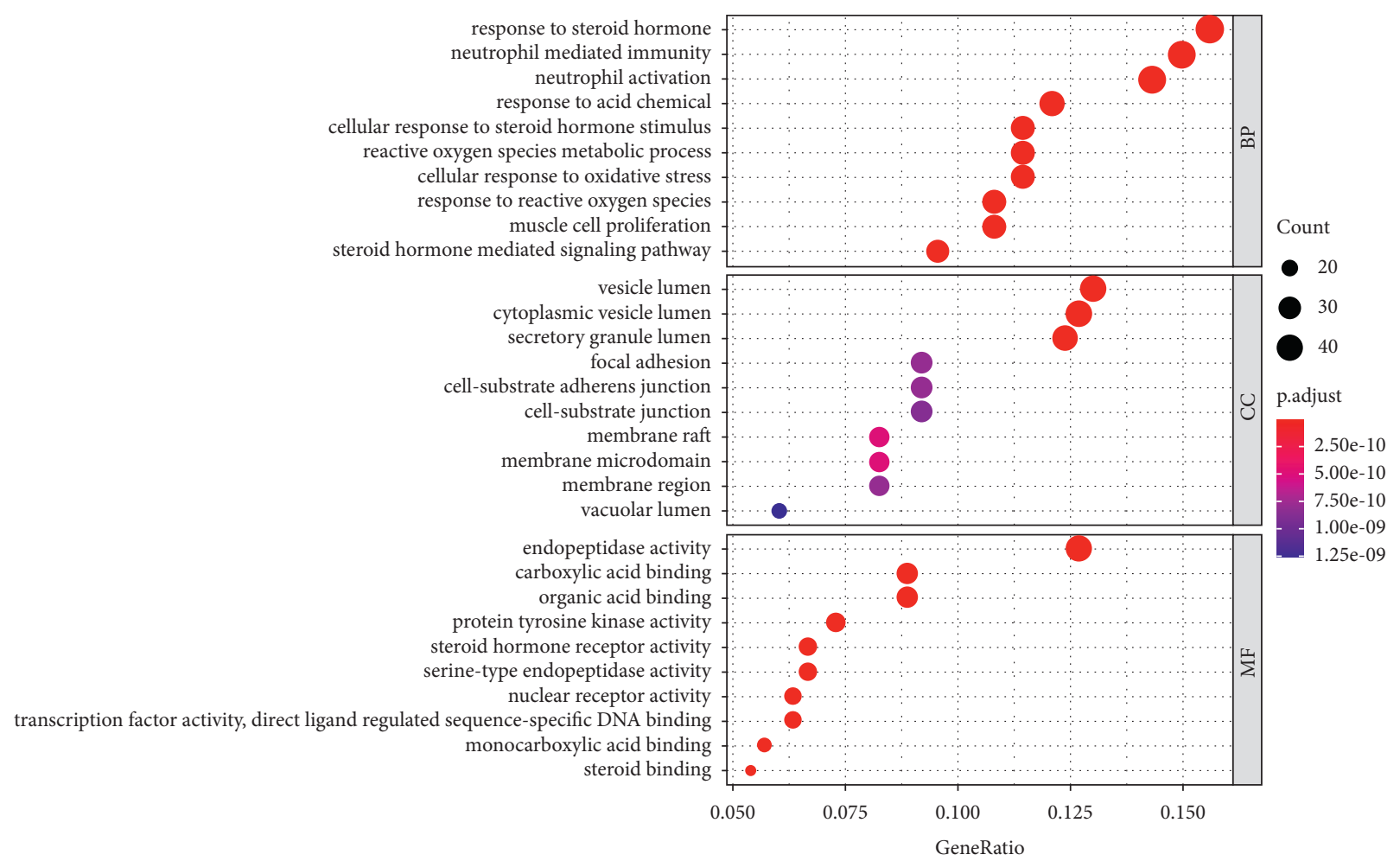

Figure 7: Functional classification of drug-disease targets by bioinformatic analysis. The biological process (BP), cellular component (CC), and molecular function (MF). Gene ratio refers to the ratio of enriched genes to all target genes, and counts refer to the number of the enriched genes. 


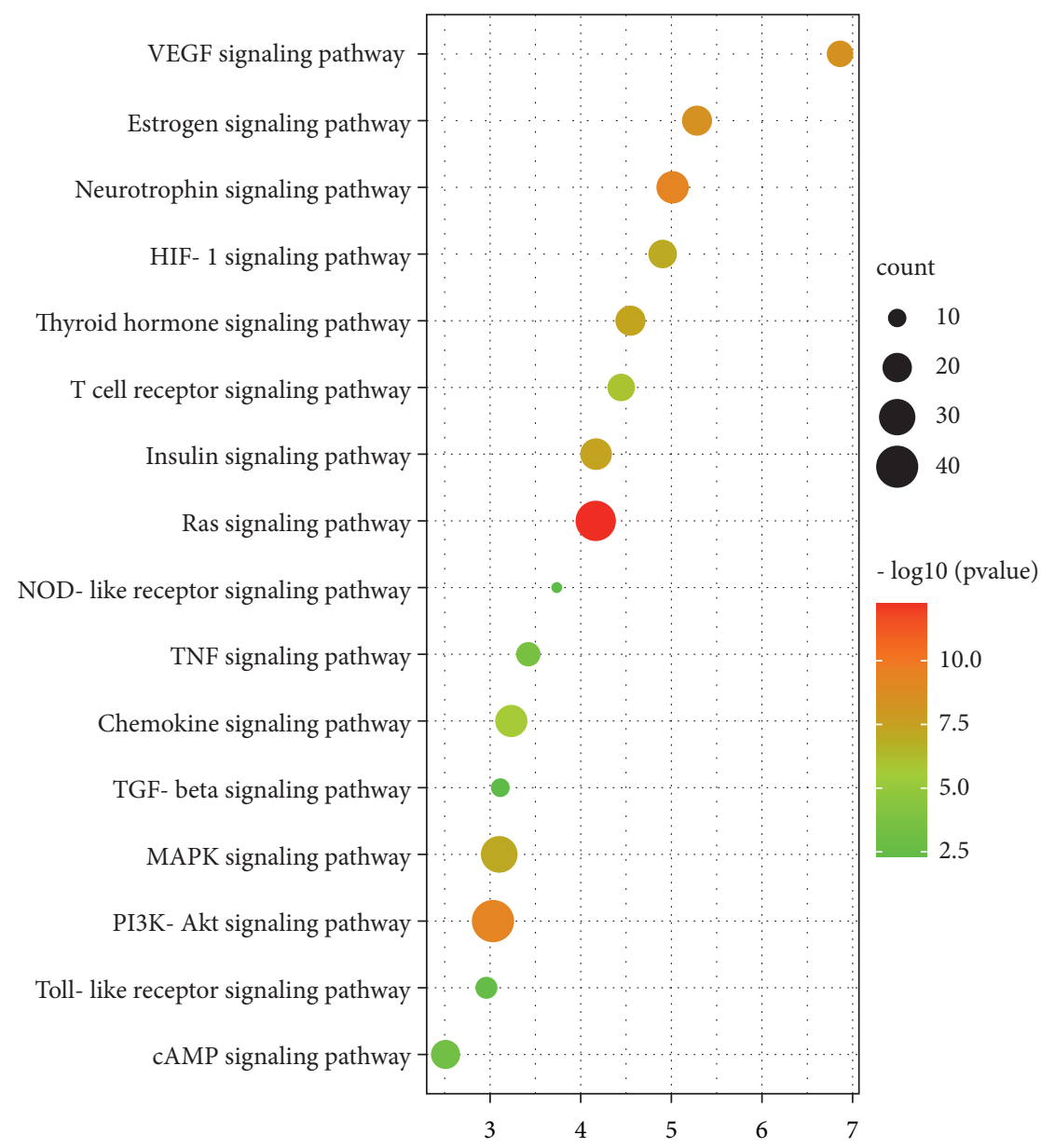

FIGURE 8: KEGG signaling pathway enrichment of screened genes. The bar chart showed the 16 pathways related to stroke. "Rich factor" represents the ratio of the number of target genes belonging to a pathway and the number of the annotated genes located in the pathway. A higher rich factor represents a higher level of enrichment. The size of the dot indicates the number of target genes in the pathway, and the color of the dot reflects the different $P$ values.

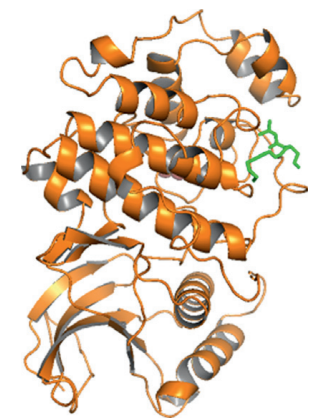

(a)

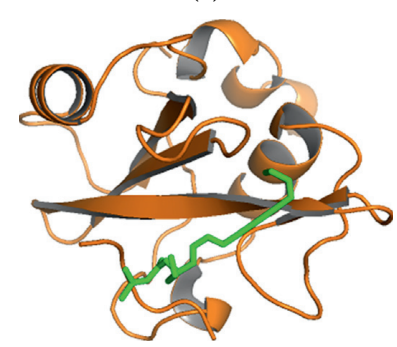

(d)

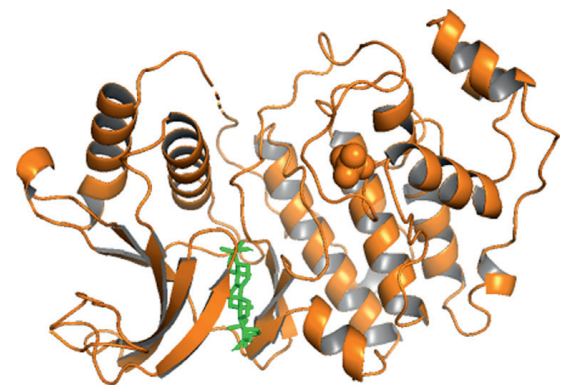

(b)

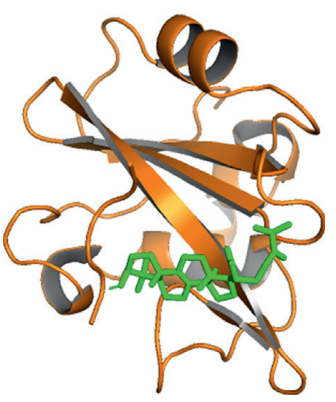

(e)

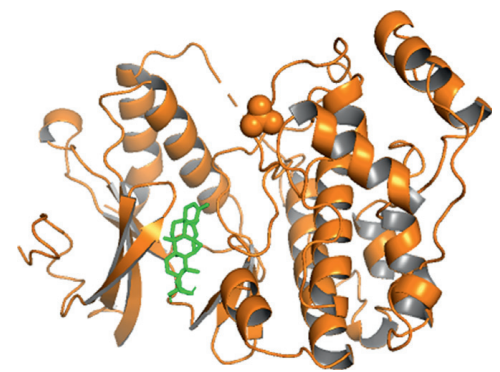

(c)

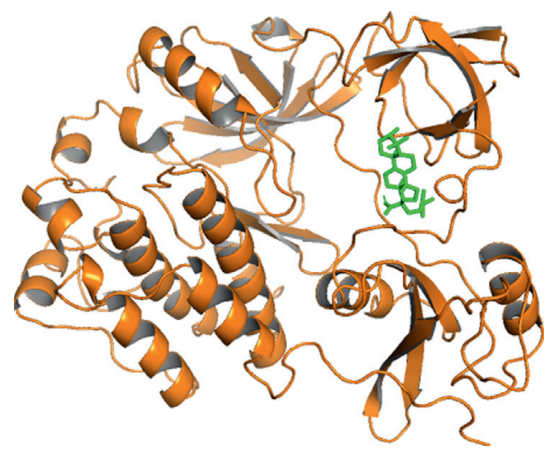

(f)

Figure 9: Continued. 


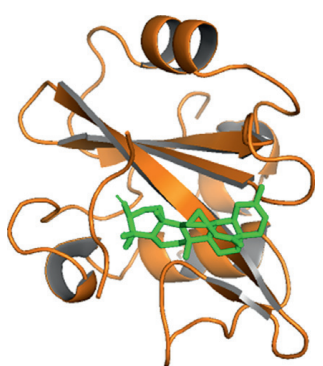

$(\mathrm{g})$

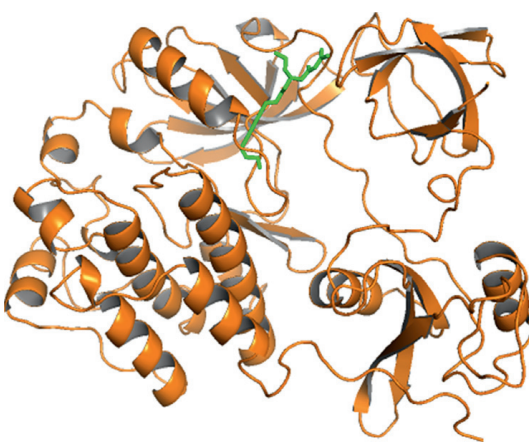

(h)

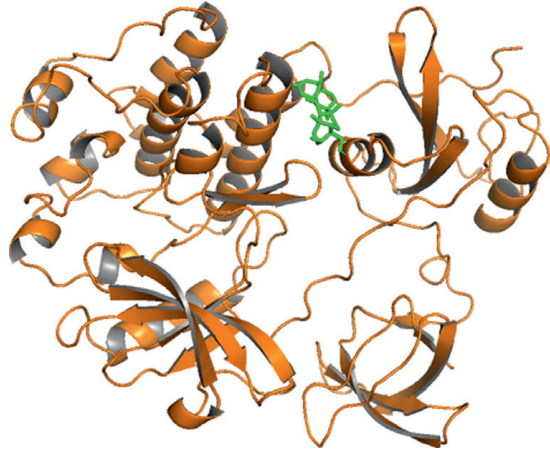

(i)

FIGURE 9: Docking results of the key ingredient and three hub target proteins. (a) 12-senecioyl-2E, 8E, 10E-atractylentriol and MAPK1; (b) eburicoic acid and MAPK1; (c) 11alpha,12alpha-epoxy-3beta-23-dihydroxy-30-nor-olean-20-en-28,12beta-olide and MAPK1; (d) 12senecioyl-2E, 8E, 10E-atractylentriol and PI3K; (e) eburicoic acid and PI3K; (f) 11alpha,12alpha-epoxy-3beta-23-dihydroxy-30-nor-olean20-en-28,12beta-olide and PI3K; (g) 12-senecioyl-2E, 8E, 10E-atractylentriol and SRC; (h) eburicoic acid and SRC; (i) 11alpha,12alphaepoxy-3beta-23-dihydroxy-30-nor-olean-20-en-28,12beta-olide and SRC.

TABLE 5: Binding energy between the key ingredients and target proteins.

\begin{tabular}{|c|c|c|c|c|}
\hline NO. & Receptor protein & Molecule ID & Ligand component & $\begin{array}{l}\text { Binding energy } \\
(\mathrm{kcal} \cdot \mathrm{mol}-1)\end{array}$ \\
\hline 1 & MAPK1 & MOL000020 & 12-Senecioyl-2E, 8E, 10E-atractylentriol & -5.99 \\
\hline 2 & MAPK1 & MOL000287 & Eburicoic acid & -8.64 \\
\hline 3 & MAPK1 & MOL001910 & $\begin{array}{l}\text { 11alpha,12alpha-epoxy-3beta-23-Dihydroxy-30-nor-olean-20-en-28, } \\
\text { 12beta-olide }\end{array}$ & -9.32 \\
\hline 4 & PIK3R1 & MOL000020 & 12-Senecioyl-2E, $8 \mathrm{E}, 10 \mathrm{E}$-atractylentriol & -6.66 \\
\hline 5 & PIK3R1 & MOL000287 & Eburicoic acid & -9.24 \\
\hline 6 & PIK3R1 & MOL001910 & $\begin{array}{l}\text { 11alpha,12alpha-epoxy-3beta-23-Dihydroxy-30-nor-olean-20-en-28, } \\
\text { 12beta-olide }\end{array}$ & -10.18 \\
\hline 7 & SRC & MOL000020 & 12-Senecioyl-2E, $8 \mathrm{E}, 10 \mathrm{E}$-atractylentriol & -7.57 \\
\hline 8 & SRC & MOL000287 & Eburicoic acid & -9.42 \\
\hline 9 & SRC & MOL001910 & $\begin{array}{l}\text { 11alpha,12alpha-epoxy-3beta-23-Dihydroxy-30-nor-olean-20-en-28, } \\
\text { 12beta-olide }\end{array}$ & -9 \\
\hline
\end{tabular}

\section{Discussion}

In this study, the anti-IS mechanism underlying the effect of DSS was explored using network pharmacology through data mining and subsequent computational modeling. It was noteworthy that our findings partially elucidate the complex anti-IS mechanism in the DSS, and they provide insight into the integrated understanding of the therapeutic efficacy and pharmaceutical activity of DSS. First, a total of 534 active ingredients were extracted from DSS. The possible targets of DSS were mined using the TCMSP database. PPI data were used to construct the core PPI network. Then, 30 candidate anti-IS targets of DSS were identified as the pivotal hub genes in the core PPI network according to the specific topological importance. In addition, GO and KEGG pathway analyses were carried out to demonstrate the candidate target biological significance after the pivotal hub genes were incorporated into ClueGO. Finally, we evaluate the neuroprotection role of DSS in IS stroke mice and detected the effect of DSS on the major drug targets in IS treatment using the Western blot.
4.1. DSS May Contribute to the Treatment of IS by Diminishing Apoptosis, Reducing Inflammation, and Oxidative Stress at Acute Stage. Cerebral ischemia-reperfusion injury induces a complex pathophysiological cascade that includes a wide range of aberrant cellular processes [30]. In the ischemic phase, reduced blood supply rapidly leads to failure of ionic gradients, excitotoxicity, and neuronal death. During the reperfusion phase, the return of oxygen contributes to oxidative stress, and the restoration of blood introduces factors that promote inflammation and edema, thereby further increasing the vulnerability of the affected tissue to death [30].

The increasing number of reports shows that apoptosis may contribute to a significant proportion of neuron death following IS [31]. Mechanistic studies have established that decreasing pro-apoptotic proteins or enhancing pro-survival proteins protects the brain after cerebral ischemia. We found that MAPK1 is the key target of DSS in the treatment of IS, and AKT1, GSK3, CASP3, and BCL2 are also among the targets. MAPK includes the extracellular signal-regulated kinase 1/2 (ERK1/2) subfamily. The phosphatidylinositol 3- 


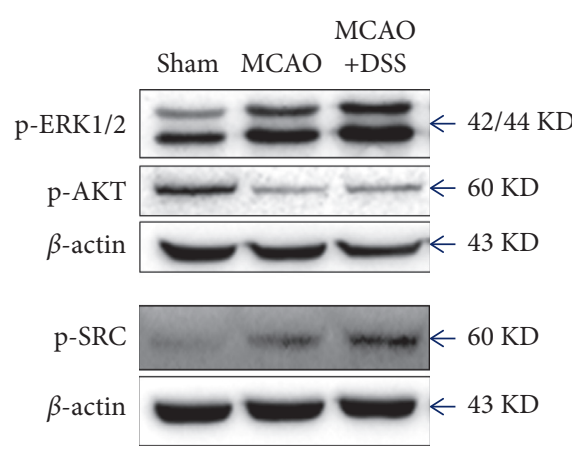

(a)

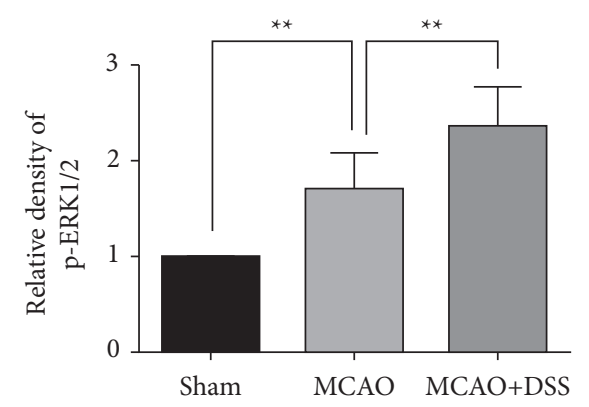

(b)

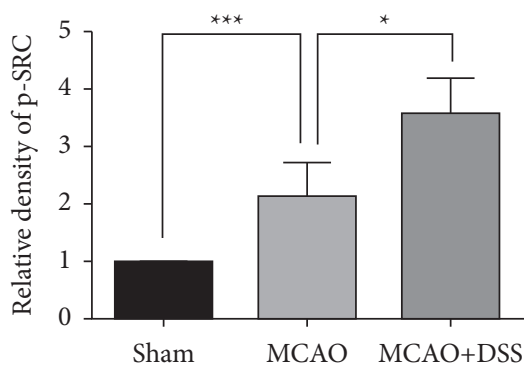

(d)

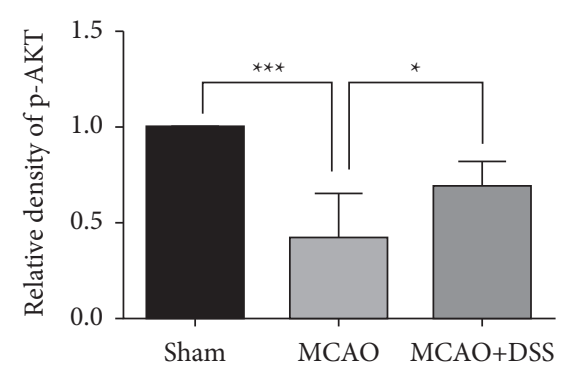

(c)

FIGURE 10: Expression of protein detected through the Western blot. (a) Representative Western blots of p-ERK1/2 and p-AKT at day 1 after MCAO surgery and the levels of p-SRC at day 7 after MCAO surgery. (b-d) Quantification of p-ERK1/2, p-AKT, and p-SRC, respectively. Values are mean $\pm \mathrm{SD} .{ }^{*} P<0.05,{ }^{* *} P<0.01,{ }^{* * *} P<0.001 . N=7$ per group.

kinase/Akt (PI3K/Akt) is an important signaling pathway involved in the regulation of cell apoptosis after stroke [32]. Many studies have shown that the activation of AKT and ERK1/2 can effectively inhibit apoptosis by regulating the expression of the anti-apoptosis gene Bcl-2 [33, 34]. Activated Akt promotes cell survival and suppresses apoptosis partly via inhibiting glycogen synthase kinase- $3 \beta$ (GSK $3 \beta$ ), an apoptosis-related molecule [31]. Caspase- 3 is considered to play an executing role at the final step of apoptosisproducing DNA fragmentation [31]. The report showed that DSS reduced the expression of caspase- 3 and upregulated the expression of $\mathrm{Bcl}-2$ at the MCAO rat model [11]. In this study, the Western blot results revealed that DSS significantly regulated the expression of $\mathrm{p}-\mathrm{ERK} 1 / 2, \mathrm{p}-\mathrm{AKT}$, and $\mathrm{Bcl}-2$. MAPK1 (ERK1/2) and PI3K, the two key targets, created 3D graphs of docking and analysis. Docking their crystal structures with the compounds, we showed that the two genes could be attached to the active pocket of the protein.

Inflammation after cerebral ischemia is a complicated pathological process starting from the activation of microglia, circulating leukocyte infiltrate (such as neutrophils, lymphocytes, and macrophages), and releasing of pro-inflammatory mediators mediated by ischemic cells and immune cells $[30,35]$. Our GO analysis showed that DSS regulated neutrophil activation and neutrophil mediated immunity. Experimental models have shown that the inhibition of neutrophilic inflammatory mechanisms reduces neurodegeneration and improves functional outcome after cerebral ischemia [36]. Based on the KEGG pathway analysis, DSS influences TNF signaling pathway. TNF signaling pathway mediates a wide range of cellular processes including inflammation, proliferation, cell migration, apoptosis, and necrosis [37]. Studies confirm that the inhibition of TNF signaling pathway might be associated with reducing neuroinflammation in IS [38].

An imbalance between free radical production and antioxidant activity leads to oxidative stress, which is a major pathologic mechanism of secondary brain damage after cerebral ischemia [30]. Our GO analysis showed that the mechanism of DSS in the treatment of IS was related to cellular response to oxidative stress and reactive oxygen species metabolic process. Superoxide dismutase 2 (SOD2) and peroxisome proliferator-activated receptor alpha (PPARA) are the core targets of DSS in IS treatment. SOD2 is an enzymatic antioxidant that catalyzes the conversion of $\mathrm{H} 2 \mathrm{O} 2$ and helps maintain the redox balance by diffusing the superoxide. Therapeutically increasing the levels of SOD2 could be an important treatment strategy in oxidative stressinduced pathology [39]. PPARA is a member of the PPAR nuclear receptor subfamily. Several studies have demonstrated that PPARA-mediated reduction in oxidative stress correlates with improved outcomes in rodent stroke models [40]. Recently, the study showed that DSS could attenuate oxidative stress against cerebral ischemic-reperfusion injury via SIRT1-dependent manner [11].

4.2. DSS May Contribute to the Treatment of IS by Increased Angiogenesis and Neurogenesis at Chronic Stage. Spontaneous neurogenesis and angiogenesis in the postacute phase are highly coordinated responses and may 
contribute to the improvement in neurologic function after stroke. Accumulating experimental studies showed that promoting post-ischemic angiogenesis and neurogenesis can improve neurological function [41]. Ischemic stroke promotes neurogenesis by several growth factors including FGF-2, IGF-1, BDNF, VEGF, and chemokines including SDF-1 and MCP-1. Stroke-induced angiogenesis is similarly regulated by many factors most notably eNOS and CSE, VEGF/VEGFR2, and Ang-1/Tie2 [41]. KEGG enrichment analysis showed that VEGF signaling pathway and FGF signaling pathway were part of the ten major signaling pathways, suggesting that the mechanisms of DSS in the treatment of IS were related to the angiogenesis. SRC is the top three targets of DSS in the IS treatment. SRC is the downstream of VEGF to regulate angiogenesis. Our Western blot analysis confirmed that DSS significantly modulated the activity of SRC. We found that IGF-1, FGFR2, NOX3 (eNOS), MMP13, and EIF4E are the key targets of DSS in the treatment of IS. VEGF confers neuroprotection and promotes neurogenesis and cerebral angiogenesis after ischemic stroke [42]. VEGF signaling promotes endothelial nitric oxide synthase (eNOS) phosphorylation and is related to the angiogenesis [43]. Our previous study showed that DSS treatment promoted focal angiogenesis at 14 days after MCAO. Our previous study showed that DSS promotes angiogenesis and neurogenesis in rat following MCAO via upregulation of VEGF protein expression and increased eNOS activity [14]. The above studies have shown that DSS can not only inhibit apoptosis, inflammatory response, and oxidative stress in the acute phase of stroke but also improve neurological function by increasing neurogenesis and angiogenesis in the chronic recovery period. It suggests that DSS can be used in any period of stroke.

4.3. DSS May Contribute to the Treatment of IS by Modulate Estrogen Level. DSS is a famous herbal formulary from the Synopsis of the Golden Chamber, which was used to treat gynecological disease, especially female abdominal pain. At present, DSS is widely used in neurological diseases [7, 14]. Therefore, it is speculated that DSS might be able to regulate estrogen levels. Both GO and KEGG results showed that DSS responds to steroid hormone and regulates the estrogen signaling pathway. A number of evidence shows that the biological effects of estrogen extend beyond the gonads to other body systems, including the brain and behavior [44]. The results of preclinical studies have shown that estrogen has neuroprotective effects in various experimental models of IS [44]. Many different biological effects of estrogen are modulated by estrogen receptor (ESR), e.g., ESR1 and ESR2. Our result showed that ESR1 is a major target of DSS for IS treatment. The reports showed that estrogen reduces the ischemic oxidative damage via an ESR1-mediated inhibition of NADPH oxidase activation [45]. Dubal et al. showed that ESR1-/-mice have more severe brain damage after MCAO [46]. The above results might in part explain why DSS can treat both gynecological diseases and stroke.

Despite abundant new findings in this study, some limitations still exist. First, the corresponding experimental validation covered only a small number of mechanisms. Second, the compounds, targets, and pathways contained in these databases may not be exhaustive. In conclusion, the study, combined with network pharmacology, molecular docking, and animal experiments, offers the systematic mechanism of DSS in the treatment of IS and clearly clarifies the synergy mechanism of DSS multicomponent and multitarget. DSS may contribute to the treatment of IS by diminishing apoptosis, reducing inflammation and oxidative stress, increasing angiogenesis and neurogenesis, and modulating estrogen level. Our results indicate that DSS can not only reduce brain injury in the acute phase of stroke but also promote neurological recovery in the chronic phase of stroke.

\section{Data Availability}

The data that support the findings of this study are available from the corresponding author upon reasonable request.

\section{Conflicts of Interest}

The authors declare that there are no conflicts of interest regarding the publication of this article.

\section{Authors' Contributions}

C. R. and Y. Y. conceived and designed the study. S.L. developed the methodology. S.L., J.X, and W.Z. performed the data acquisition. C. R. performed data analysis and interpretation. S. L., C.G., H. L., W.Y., and W.Z did animal experiments and molecular biology experiments. C. R. drafted, reviewed, and revised the manuscript. All authors read and approved the final manuscript.

\section{Acknowledgments}

This work was supported by the National Natural Science Foundation of China (Grant Nos. 81971114, 81573867, 81801313, and 81801142) and the National Key R\&D Program of China (2017YFC1308402).

\section{References}

[1] V. L. Feigin, G. Nguyen, K. Cercy et al., "Global, regional, and country-specific lifetime risks of stroke, 1990 and 2016," New England Journal of Medicine, vol. 379, no. 25, pp. 2429-2437, 2018.

[2] GBD 2019 Diseases and Injuries Collaborators, "Global burden of 369 diseases and injuries in 204 countries and territories, 1990-2019: a systematic analysis for the Global Burden of Disease Study 2019," Lancet (London, England), vol. 396, pp. 1204-1222, 2020.

[3] W. J. Powers, A. A. Rabinstein, T. Ackerson et al., "Guidelines for the early management of patients with acute ischemic stroke: 2019 update to the 2018 guidelines for the early management of acute ischemic stroke: a guideline for Healthcare professionals from the American heart association/American stroke association," Stroke, vol. 50, no. 12, pp. e344-e418, 2019. 
[4] M. Goyal, B. K. Menon, W. H. van Zwam et al., "Endovascular thrombectomy after large-vessel ischaemic stroke: a metaanalysis of individual patient data from five randomized trials," The Lancet, vol. 387, no. 10029, pp. 1723-1731, 2016.

[5] X. Ji, W. Zhao, C. Wu et al., "Multiphase adjuvant neuroprotection: a novel paradigm for improving acute ischemic stroke outcomes," Brain circulation, vol. 6, no. 1, pp. 11-18, 2020.

[6] Z. Xu, "Modernization: one step at a time," Nature, vol. 480, no. 7378, pp. S90-S92, 2011.

[7] T. Itoh, S. Michijiri, S. Murai et al., "Regulatory effect of Danggui-Shaoyao-San on central cholinergic nervous system dysfunction in mice," The American Journal of Chinese Medicine, vol. 24, no. 03n04, pp. 205-217, 1996.

[8] L. Chen, J. Qi, Y.-X. Chang, D. Zhu, and B. Yu, "Identification and determination of the major constituents in Traditional Chinese Medicinal formula Danggui-Shaoyao-San by HPLCDAD-ESI-MS/MS," Journal of Pharmaceutical and Biomedical Analysis, vol. 50, no. 2, pp. 127-137, 2009.

[9] X. Fu, Q. Wang, Z. Wang, H. Kuang, and P. Jiang, "DangguiShaoyao-San: new hope for Alzheimer's disease," Aging and Disease, vol. 7, no. 4, pp. 502-513, 2016.

[10] Y. Kitabayashi, K. Shibata, T. Nakamae, J. Narumoto, and K. Fukui, "Effect of traditional Japanese herbal medicine TokiShakuyaku-San for mild cognitive impairment: SPECT study," Psychiatry and Clinical Neurosciences, vol. 61, no. 4, pp. 447-448, 2007.

[11] Y. Luo, H. Chen, B. Tsoi, Q. Wang, and J. Shen, "DangguiShaoyao-San (DSS) ameliorates cerebral ischemia-reperfusion injury via activating SIRT1 signaling and inhibiting NADPH oxidases," Frontiers in Pharmacology, vol. 12, Article ID 653795, 2021.

[12] Y. Ren, Y. Zhao, and S. Lu, "Clinical observation on effect of Danggui Shaoyao powder Combined with rehabilitation technique in treatment of 60 cases cerebral infarction," Liaoning University Traditional Chinese Medical, vol. 087, no. 8, 2013.

[13] I. Hatip-Al-Khatib, F. B. Hatip, Y. Yoshimitsu et al., "Effect of Toki-Shakuyaku-san on acetylcholine level and blood flow in dorsal hippocampus of intact and twice-repeated ischemic rats," Phytotherapy Research, vol. 21, no. 3, pp. 291-294, 2007.

[14] C. Ren, B. Wang, N. Li, K. Jin, and X. Ji, "Herbal formula Danggui-Shaoyao-San promotes neurogenesis and angiogenesis in rat following middle cerebral artery occlusion," Aging and Disease, vol. 6, no. 4, pp. 245-253, 2015.

[15] J. Qiu, "A culture in the balance," Nature, vol. 448, no. 7150, pp. 126-128, 2007.

[16] F. Cheng, I. A. Kovács, and A. L. Barabási, "Network-based prediction of drug combinations," Nature Communications, vol. 10, p. 1197, 2019.

[17] J. Zhao, P. Jiang, and W. Zhang, "Molecular networks for the study of TCM pharmacology," Briefings in Bioinformatics, vol. 11, no. 4, pp. 417-430, 2010.

[18] J. Ru, P. Li, J. Wang et al., "TCMSP: a database of systems pharmacology for drug discovery from herbal medicines," Journal of Cheminformatics, vol. 6, p. 13, 2014.

[19] X. Wang, Y. Shen, S. Wang et al., "PharmMapper 2017 update: a web server for potential drug target identification with a comprehensive target pharmacophore database," Nucleic Acids Research, vol. 45, no. W1, pp. W356-w360, 2017.

[20] A. P. Davis, T. C. Wiegers, J. Wiegers et al., "CTD Anatomy: analyzing chemical-induced phenotypes and exposures from an anatomical perspective, with implications for environmental health studies," Current Research in Toxicology, vol. 2, pp. 128-139, 2021.

[21] Y. Wang, S. Zhang, F. Li et al., "Therapeutic target database 2020: enriched resource for facilitating research and early development of targeted therapeutics," Nucleic Acids Research, vol. 48, pp. D1031-d1041, 2020.

[22] D. Szklarczyk, J. H. Morris, H. Cook et al., "The STRING database in 2017: quality-controlled protein-protein association networks, made broadly accessible," Nucleic Acids Research, vol. 45, no. D1, pp. D362-d368, 2017.

[23] C. H. Chin, S. H. Chen, H. H. Wu, C. W. Ho, M. T. Ko, and C. Y. Lin, "CytoHubba: identifying hub objects and subnetworks from complex interactome," BMC Systems Biology, vol. 8, no. Suppl 4, 2014.

[24] X. Jiao, B. T. Sherman, D. W. Huang et al., "DAVID-WS: a stateful web service to facilitate gene/protein list analysis," Bioinformatics, vol. 28, no. 13, pp. 1805-1806, 2012.

[25] M. Kanehisa, M. Araki, S. Goto et al., "KEGG for linking genomes to life and the environment," Nucleic Acids Research, vol. 36, no. Database, pp. D480-D484, 2008.

[26] O. Trott and A. J. Olson, "AutoDock Vina: improving the speed and accuracy of docking with a new scoring function, efficient optimization, and multithreading," Journal of Computational Chemistry, vol. 31, pp. 455-461, 2010.

[27] J. Chang, L. Liu, Y. Wang, Y. Sui, H. Li, and L. Feng, "Investigating the multitarget mechanism of traditional Chinese medicine prescription for cancer-related pain by using network pharmacology and molecular docking approach," Evidence-Based Complementary and Alternative Medicine, vol. 2020, Article ID 7617261, 11 pages, 2020.

[28] X. Ji, C. Ren, S. Li et al., "Enhanced oxidative stress response and neuroprotection of combined limb remote ischemic conditioning and atorvastatin after transient ischemic stroke in rats," Brain Circulation, vol. 3, no. 4, pp. 204-212, 2017.

[29] C. Ren, Y. Liu, C. Stone et al., "Limb remote ischemic conditioning ameliorates cognitive impairment in rats with chronic cerebral hypoperfusion by regulating glucose transport," Aging and Disease, vol. 12, no. 5, pp. 1197-1210, 2021.

[30] G. Li, K. C. Morris-Blanco, M. S. Lopez et al., "Impact of microRNAs on ischemic stroke: from pre- to post-disease," Progress in Neurobiology, vol. 163-164, pp. 59-78, 2018.

[31] D. Radak, N. Katsiki, I. Resanovic et al., "Apoptosis and acute brain ischemia in ischemic stroke," Current Vascular Pharmacology, vol. 15, no. 2, pp. 115-122, 2017.

[32] U. Kilic, A. B. Caglayan, M. C. Beker et al., "Particular phosphorylation of PI3K/Akt on Thr308 via PDK-1 and PTEN mediates melatonin's neuroprotective activity after focal cerebral ischemia in mice," Redox Biology, vol. 12, pp. 657-665, 2017.

[33] N. Sawe, G. Steinberg, and H. Zhao, "Dual roles of the MAPK/ ERK1/2 cell signaling pathway after stroke," Journal of Neuroscience Research, vol. 86, no. 8, pp. 1659-1669, 2008.

[34] E. Kilic, Ü. Kilic, J. Soliz, C. L. Bassetti, M. Gassmann, and D. M. Hermann, "Brain-derived erythropoietin protects from focal cerebral ischemia by dual activation of ERK-1/-2 and Akt pathways," The FASEB Journal, vol. 19, no. 14, pp. 2026-2028, 2005.

[35] L. Belayev, N. Bazan, A. Obenaus et al., "Blocking pro-inflammatory platelet-activating factor receptors and activating cell survival pathways: a novel therapeutic strategy in experimental ischemic stroke," Brain Circulation, vol. 6, no. 4, pp. $260-268,2020$.

[36] L. Zhang, Z. G. Zhang, R. L. Zhang, M. Lu, M. Krams, and M. Chopp, "Effects of a selective CD11b/CD18 antagonist and 
recombinant human tissue plasminogen activator treatment alone and in combination in a rat embolic model of stroke," Stroke, vol. 34, no. 7, pp. 1790-1795, 2003.

[37] J. Bradley, "TNF-mediated inflammatory disease," The Journal of Pathology, vol. 214, no. 2, pp. 149-160, 2008.

[38] M. Liguz-Lecznar, R. Zakrzewska, and M. Kossut, "Inhibition of TNF- $\alpha$ R1 signaling can rescue functional cortical plasticity impaired in early post-stroke period," Neurobiology of Aging, vol. 36, no. 10, pp. 2877-2884, 2015.

[39] S. Orellana-Urzúa, I. Rojas, L. Libran, and R. Rodrigo, "Pathophysiology of ischemic stroke: role of oxidative stress," Current Pharmaceutical Design, vol. 26, pp. 4246-4260, 2020.

[40] A. C. Boese, J.-P. Lee, and M. H. Hamblin, "Neurovascular protection by peroxisome proliferator-activated receptor $\alpha$ in ischemic stroke," Experimental Neurology, vol. 331, Article ID 113323,2020

[41] L. Ruan, B. Wang, Q. ZhuGe, and K. Jin, "Coupling of neurogenesis and angiogenesis after ischemic stroke," Brain Research, vol. 1623, pp. 166-173, 2015.

[42] D. A. Greenberg and K. Jin, "From angiogenesis to neuropathology," Nature, vol. 438, no. 7070, pp. 954-959, 2005.

[43] S. C. Bir, Y. Xiong, C. G. Kevil, and J. Luo, "Emerging role of PKA/eNOS pathway in therapeutic angiogenesis for ischaemic tissue diseases," Cardiovascular Research, vol. 95, no. 1, pp. 7-18, 2012.

[44] E. C. Koellhoffer and L. D. McCullough, "The effects of estrogen in ischemic stroke," Translational Stroke Research, vol. 4, no. 4, pp. 390-401, 2013.

[45] Q.-G. Zhang, L. Raz, R. Wang et al., "Estrogen attenuates ischemic oxidative damage via an estrogen receptor-mediated inhibition of NADPH oxidase activation," Journal of Neuroscience, vol. 29, no. 44, pp. 13823-13836, 2009.

[46] D. B. Dubal, H. Zhu, J. Yu et al., "Estrogen receptor alpha, not beta, is a critical link in estradiol-mediated protection against brain injury," Proceedings of the National Academy of Sciences, vol. 98, no. 4, pp. 1952-1957, 2001. 